\title{
DIFFERENTIAL EFFECTS OF LAVENDER AND ROSEMARY ON AROUSAL AND COGNITIVE PERFORMANCE
}

\author{
by \\ Livia Sumegi \\ BA Hons Carleton University, 2007 \\ A thesis submitted to \\ the Faculty of Graduate and Postdoctoral Affairs \\ in partial fulfillment of the requirements for the degree of \\ Master of Arts \\ in \\ Psychology
}

Carleton University

Ottawa, Canada

C2011, Livia Sumegi 


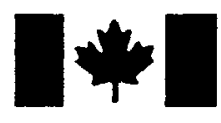

Library and Archives

Canada

Published Heritage

Branch

395 Wellington Street

Ottawa ON K1A ON4

Canada
Bibliothèque et

Archives Canada

Direction du

Patrimoine de l'édition

395 , rue Wellington

Ottawa ON K1A ON4

Canada
Your file Votre référence

ISBN: 978-0-494-79582-8

Our file Notre référence

ISBN: 978-0-494-79582-8
NOTICE:

The author has granted a nonexclusive license allowing Library and Archives Canada to reproduce, publish, archive, preserve, conserve, communicate to the public by telecommunication or on the Internet, loan, distribute and sell theses worldwide, for commercial or noncommercial purposes, in microform, paper, electronic and/or any other formats.

The author retains copyright ownership and moral rights in this thesis. Neither the thesis nor substantial extracts from it may be printed or otherwise reproduced without the author's permission.
AVIS:

L'auteur a accordé une licence non exclusive permettant à la Bibliothèque et Archives Canada de reproduire, publier, archiver, sauvegarder, conserver, transmettre au public par télécommunication ou par l'Internet, prêter, distribuer et vendre des thèses partout dans le monde, à des fins commerciales ou autres, sur support microforme, papier, électronique et/ou autres formats.

L'auteur conserve la propriété du droit d'auteur et des droits moraux qui protège cette thèse. $\mathrm{Ni}$ la thèse ni des extraits substantiels de celle-ci ne doivent être imprimés ou autrement reproduits sans son autorisation.
In compliance with the Canadian Privacy Act some supporting forms may have been removed from this thesis.

While these forms may be included in the document page count, their removal does not represent any loss of content from the thesis.
Conformément à la loi canadienne sur la protection de la vie privée, quelques formulaires secondaires ont été enlevés de cette thèse.

Bien que ces formulaires aient inclus dans la pagination, il n'y aura aucun contenu manquant.

\section{Canadä}




\begin{abstract}
This study assessed the impact of the essential oils of lavender and rosemary on cognitive performance, mood, and physiology in normal adults. Ninety participants were assigned to either one of the two scent conditions or a control condition, and subsequently performed the Cognitive Drug Research (CDR) task battery with an added arithmetic component. Heart rate, skin conductance, and EEG measures were taken throughout the experiment. Results demonstrate that certain aspects of cognitive performance and physiology can be influenced by exposure to these aromas. Some empirical substantiation regarding the purported sedating effects of lavender and stimulating effects of rosemary was presented; however, the notion of clear and opposing cognitive and physiological profiles for the aromas of lavender and rosemary received only mixed support. Physiological effects were not mirrored in self-report data which calls into question much previous research that has relied solely on self-report data when assessing physiological effects of scents.
\end{abstract}




\section{Dedication}

This thesis is dedicated to my father Zsolt Sumegi (1945-2010). 


\section{Acknowledgments}

I have had a great deal of support from many people during the writing of this thesis; foremost among them is my supervisor Dr. Gitte Lindgaard. I would like to express my appreciation and gratitude to her for all her support and guidance throughout my graduate years. Her constant mentorship has been invaluable to the completion of this thesis and the success of my graduate work. I would also like to thank Dr. Matthew Young, Dr. Alfonso Abizaid, and Dr. Alex Ramirez for serving on my committee and providing me with insightful feedback. Thanks to Etelle Bourassa, our graduate advisor, who has patiently answered all my questions and who was always ready with a word of encouragement. Finally, I would like to thank my family for their unwavering support and my partner Eric Brown for looking after everything else in my life while I wrote this thesis. 


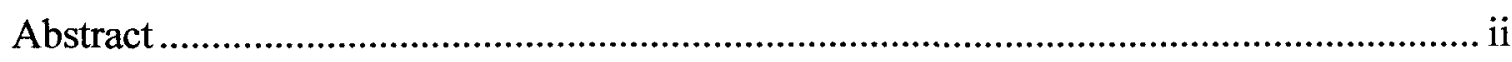

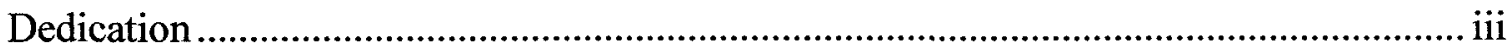

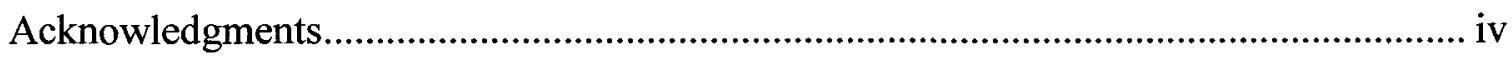

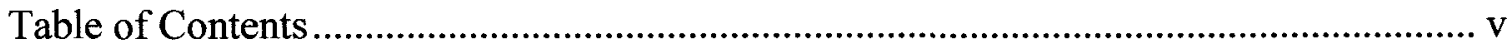

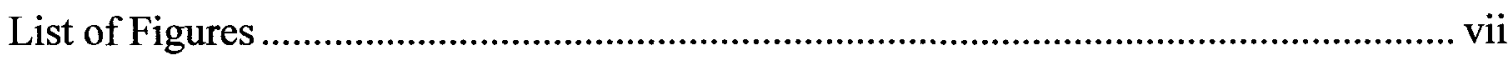

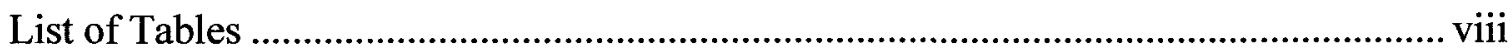

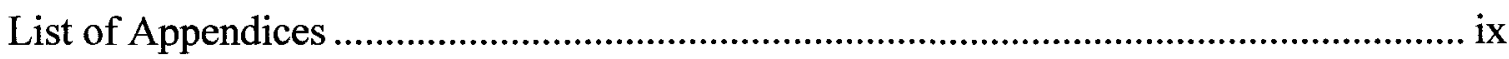

DIFFERENTIAL EFFECTS OF LAVENDER AND ROSEMARY ON COGNITIVE

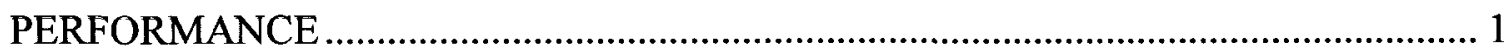

Theoretical Framework ........................................................................................ 4

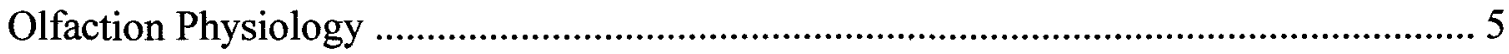

Effects of Lavender and Rosemary on Mood .................................................... 8

Psychological Effects of Lavender and Rosemary on Cognitive Tasks ........................ 10

Arithmetic Computation ............................................................. 10

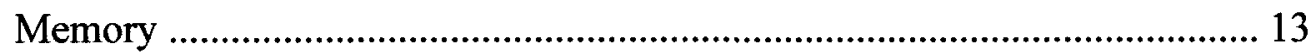

Attention/Vigilance ....................................................................... 14

Physiological Effects of Lavender and Rosemary .................................................. 18

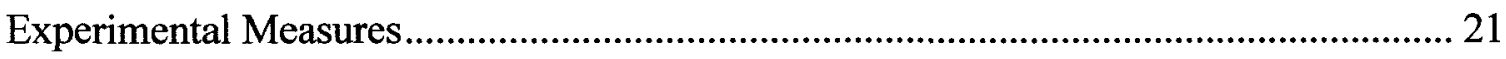

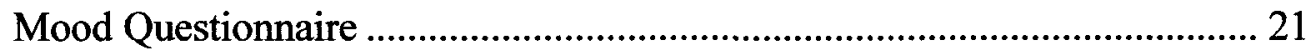

Physiological Measurements. ....................................................... 21

Arithmetic Computations .............................................................. 23

Cognitive Drug Research (CDR) Battery ........................................ 23 
Primary Cognitive Outcome Measures......................................................... 27

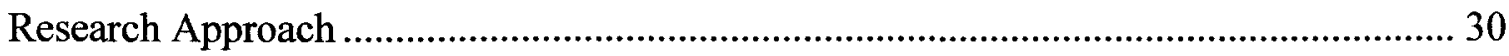

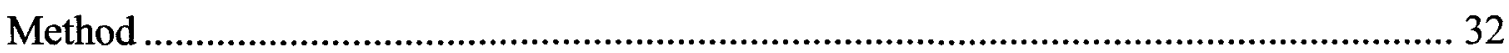

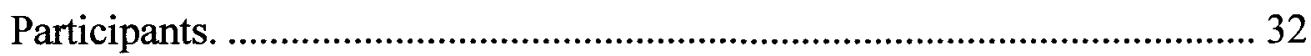

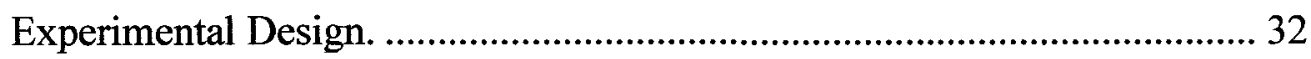

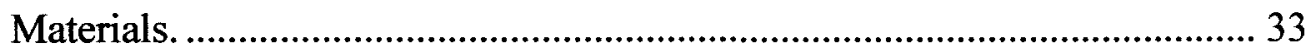

Apparatus and Data Processing. .................................................................... 34

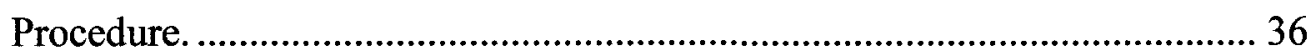

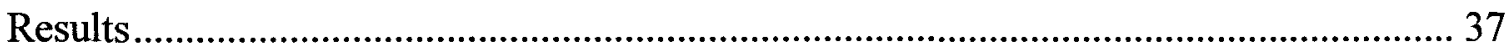

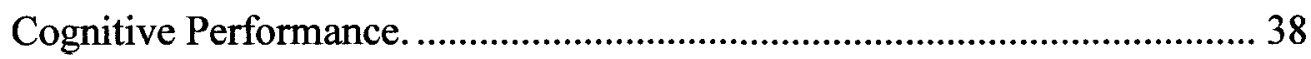

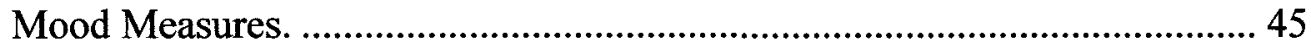

Autonomic Nervous System Parameters ...................................................... 47

Electroencephalogram (EEG) Data. .......................................................... 55

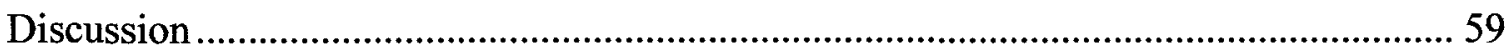

Summary of the Main Findings and Contributions. ......................................... 59

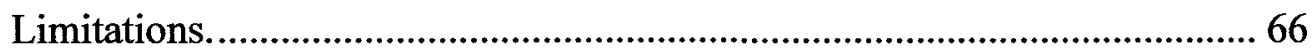

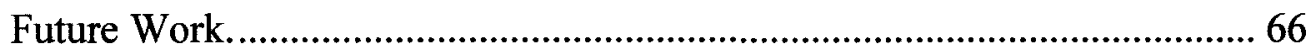

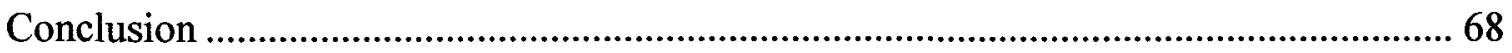

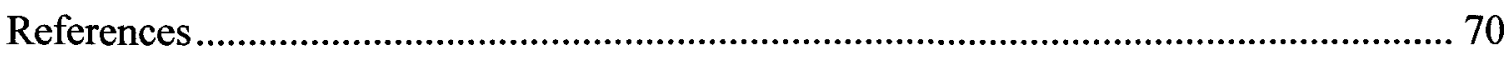

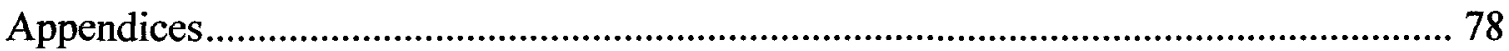




\section{List of Figures}

Figure 1. Schematic representation of the CDR battery ............................................. 29

Figure 2. Diagram illustrating the experimental design.......................................... 33

Figure 3. Effect of lavender and rosemary on the Simple Reaction Time task outcome measure of the CDR test battery.

Figure 4. Effect of lavender and rosemary on the Digit Vigilance task outcome measure

of the CDR test battery

Figure 5. Effect of lavender and rosemary on the Spatial Working Memory task outcome measure of the CDR test battery 42

Figure 6. Effect of lavender and rosemary on the Speed of Attention factor derived from the CDR test battery. 43

Figure 7. Effect of lavender and rosemary on the Speed of Memory factor derived from the CDR test battery. 44

Figure 8 . Mean heart rate by participant group, collapsed across tasks.

Figure 9. Task-related heart rate by participant group. 50

Figure 10. Task-related skin conductance levels by participant group. 54

Figure 11. A comparison of mean alpha and beta amplitudes by participant group, collapsed across tasks. 58 


\section{List of Tables}

Table 1: Descriptive Statistics for POMS subscales yielding significant main effect.......47

Table 2: For significant omnibus $\mathrm{F}$ tests, mean differences for task-related heart rate by group as identified by Bonferroni multiple comparisons .

Table 3: For significant omnibus F tests, descriptive statistics and effects of alpha amplitude power by group, as identified by the Games-Howell post hoc criterion ......... 57 


\section{List of Appendices}

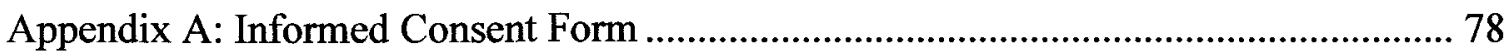

Appendix B: Pre-Experimental Questionnaire ................................................... 80

Appendix C: CDR System Task Instructions ..................................................... 81

Appendix D: Arithmetic Computations ............................................................... 85

Appendix E: Post-Experimental Questionnaire ................................................... 90

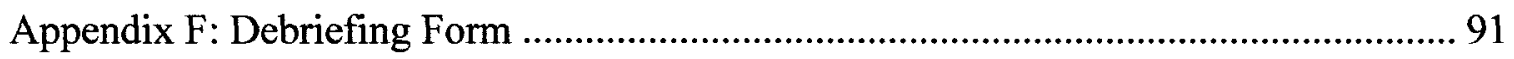

Appendix G: Consent to Use Data ................................................................. 92

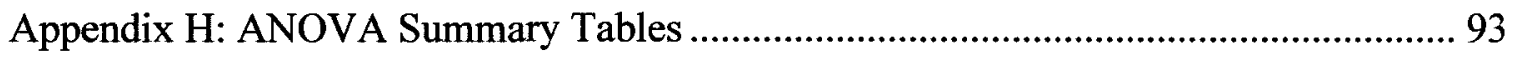

Appendix I: Comparison Table of CDR Outcome Variables by Participant Group ......... 98 


\section{DIFFERENTIAL EFFECTS OF LAVENDER AND ROSEMARY ON AROUSAL AND COGNITIVE PERFORMANCE}

Though in its infancy, scent research has begun to highlight the profound influence of scents on human psychology as well as on physiological conditions. Although prevailing wisdom holds that the sense of olfaction is simply an evolutionary vestige, scientific understanding has begun to shift such perceptions to reveal that the sense of smell elicits far-ranging and unexpected effects on both cognition and physiology (Tisserand, 1977; Moss, Cook, Wesnes, \& Duckett, 2003). Generally, the influences of aromas on humans are divided into physiological and psychological effects (Tisserand, 1977). Physiological effects directly act on the physical organism; the psychological effects act via the olfactory system, which consequently may produce a physiological effect (Hongratanaworakit, 2004).

While the influences of ambient fragrances on arousal level and cognitive task performance have been studied, assessments have been primarily based on anecdotal rather than empirical evidence (Buchbauer, Jirovetz, Jager, Dietrich, \& Plank, 1991) likely due to the inherent complexity of the sense of smell and the difficulties that accompany scent research (Shepherd, 2004). Therefore, research into the psychological and physiological effects of fragrance inhalation has put a disproportionate amount of weight on self-report data as opposed to objective measures with the result that minimal data exist regarding objective responses to fragrance. Furthermore, a lack of standardized experimental protocol means that valid and reliable empirical evidence regarding psychological and physiological responses to fragrance inhalation is scarce within the scientific community. The conclusions of 
many studies are therefore inconsistent if not ambiguous.

Although the literature concerned with psychological and physiological effects of fragrances is limited, much effort has gone into investigating the influence of the essential oils of lavender and rosemary on cognitive performance and physiology. These two particular fragrances are of interest as they are believed to elicit opposite physiological reactions. While the inhalation of lavender is largely reputed as eliciting a sedative effect (Torii et al., 1988; Buchbauer et al., 1991; Klemm, Lute, Hendrix, \& Warrenburg, 1992; Wolfe \& Herzberg, 1996; D’Angelo, 2002; Raudenbush, Koon, Smith, \& Zoladz, 2003), rosemary allegedly evokes arousal (Kovar, Gropper, Friess, \& Ammon, 1987; Diego, et al., 1998; Saeki \& Shiohara, 2001; Moss et al., 2003). Arousal has been conceptualized along with valence as the two fundamental dimensions of emotion (Russell, 1980; Lang, 1995). In this respect, valence is regarded as a linear continuum, specifying the extent to which an experience is positive or negative/pleasant or unpleasant, whereby stimuli of low (more negative) valence represents a less pleasant sensory experience than those of higher (more positive) valence (Winston et al., 2005). Arousal is purported to be orthogonal to valence and refers to the intensity of the emotional activation, extending from an unaroused calm state to a state of high excitation or agitation (Mehrabian \& Russell, 1974; Russell, 1980; Lang, Greenwald, Bradley, \& Hamm, 1993). Studies have attempted to evoke affective states in experimental settings by having participants rate pictures (Lang et al., 1998), words (Bradley \& Lang, 1999a), sounds (Bradley \& Lang, 1999b), and odours (Bensafi et al., 2001) for pleasure and arousal. Similar patterns were reported across all sensory modalities in that arousal levels increase with 
emotional valence (either positive or negative). For items rated as neutral in valence, arousal ratings did not reach the high levels that were associated with either pleasant or unpleasant stimuli (Bensafi et al., 2002). It is widely accepted that affective experience can result with very minimal cognitive involvement or awareness (AlaouiIsmaili, Robin, Rada, Dittmar, \& Vernet-Maury, 1997) and, therefore, subjective selfreport data may not be representative of the psycho-physiological reactions that manifest from emotional activation. Therefore, aside from these subjective evaluations, arousal has also been measured by way of objective variations in the autonomic nervous system; these variations have also been associated with hedonic valence. For example, physiological measurements of arousal such as heart rate variation, have been shown to be moderated by pleasantness, where unpleasant odours generally increase heart rate and pleasant ones decrease it (Brauchli, Ruegg, Etzweiler, \& Zeier, 1995; Alaoui-Ismaili et al., 1997). In the olfactory sensory modality, it has been demonstrated that odours can induce both positive and negative emotional experiences, influencing mood, cognition, behaviour, autonomic nervous system parameters, as well as cerebral activity (Bensafi et al., 2001).

Due to the interest and controversy surrounding the alleged opposite arousal responses elicited by inhaling lavender and rosemary, the effects of these two aromas on arousal and cognitive performance were examined in the present study. If the opposing responses of lavender and rosemary were objectively established, it would be of interest to the scientific study of the impact of aromas on various psychophysiological parameters since they are believed to represent physiological extremes and would therefore provide a good model of the potential changes evoked in humans 
as a result of their exposure to these and conceivably other odorants. Arousal levels were psychologically assessed using self-report questionnaires and were physiologically evaluated by measuring autonomic nervous system and cerebral activity.

Therefore, this study will also enable comparisons to be drawn between participants' subjective self-reported feelings of arousal with their objective physiological expressions of arousal. Disparity between these subjective and objective measures brings into question much of the past research in this field that has relied solely on participants' self-reported reactions to fragrance inhalation. Considering the influence of emotion and hedonic valence on arousal, the present study included self evaluations of odour pleasantness with those of mood and arousal in addition to an array of electrophysiological measurements to elucidate whether the effects are mediated by the subjective experience of the odour. Cognitive task performance was assessed using the Cognitive Drug Research (CDR) computerized assessment battery to investigate the influence of the scents on various cognitive domains as some areas may be more sensitive to these effects than others.

\section{Theoretical Framework}

The theoretical basis for the present research is rooted in the Yerkes-Dodson law of arousal. This widely respected theory supposes an empirical relationship between arousal and performance. This process is graphically illustrated as an inverted U-shaped function between arousal and performance. In accordance with this theory, there is an optimal arousal level (peak of the inverted-U curve) above or below which performance decreases (Yerkes \& Dodson, 1908). When applying the 
Yerkes-Dodson theory to the present research, above this point of optimal arousal, a stimulating odour would decrease performance whereas a sedating one would increase it. Below this optimum, the odours would elicit an opposite effect. Therefore, it is assumed that sedating odours such as lavender are more effective than stimulating odours for demanding/stressful tasks because of the anxiolitic effect they elicit. Conversely, stimulating odours such as rosemary are more effective for less challenging or monotonous tasks as they help to maintain alertness (Shimizu et al., 2008). The Yerkes-Dodson law provides a theoretical foundation for the relationship observed between arousal and performance and, based on basic human physiology, science can theoretically demonstrate that fragrance molecules can excite or inhibit particular pathways in the brain, leading to feelings of arousal and subsequent changes in cognition. To evaluate the effects of the aromas of lavender and rosemary, the present research focused on participants' physiological manifestations of the aroma exposure as well as any accompanying changes to cognitive performance. This thesis first introduces olfaction physiology as it relates to neurobiological understandings of cognition and unconscious physiological processes that underlie mood and behaviour. The following sections will provide a critical review of studies testing the influence of lavender and rosemary on various cognitive tasks as well as physiological dimensions.

\section{Olfaction Physiology}

Olfaction is considered to be phylogenetically older than any other sensory system; nevertheless, it remains the least understood of the sensory modalities (Philpott, Bennett, \& Murty, 2008). With approximately a thousand different kinds of olfactory 
receptors in our nose, the olfactory system is highly discriminatory, enabling humans to register as many as ten thousand different fragrances (Lis-Balchin, 2006). The range and complexity of this system is highlighted when compared to vision, for example, in which there are only four kinds of receptors - red, green, and blue cones plus rods (Kaye, 2004). Further, the sense of smell in humans is estimated to be approximately ten thousand times more sensitive than our other senses (Damian \& Damian, 1995).

With the inhalation of a scent, odorant molecules travel to the roof of the nasal cavity and dissolve in the olfactory epithelium, a fine mucous lining which consists of millions of olfactory receptor neurons (Zillmer \& Spiers, 2001). Each of the millions of receptor neurons send processes down to the surface of the nasal mucosa which divide into cilia (hairlike filaments). Cilia are in fact exposed extensions of the brain which extend directly into the atmosphere. Olfaction is, therefore, the most direct interface between the brain and the outside environment (Damian \& Damian, 1995). The reception of olfactory stimuli occurs in these cilia, where the ciliary receptors detect the aromatic molecules dissolved in the air which bind to specific receptors on the surface of the cilia. The axons of the olfactory receptors project through perforations in the thin cribriform plate of the skull and synapse with the olfactory bulb. When comparing it to other sensory systems, the olfactory system presents several originalities primarily due to its direct access to the brain - taste, touch, hearing and sight reach the brain through indirect pathways (Millot, Brand, \& Morand, 2002; Lis-Balchin, 2006). Specifically, all other sensory systems first pass through the thalamus and then onto the neocortex; however, from the olfactory bulb, the primary projections of the olfactory system first innervate the primitive limbic system directly through the amygdala and hippocampus formation 
before passing through the thalamus and onto the frontal cortex and other areas of the neocortex. Therefore, this unique and direct connection of the olfactory system to the limbic system results in an instantaneous effect of scent on mood and emotion (Zillmer \& Spiers, 2001).

With respect to evolutionary development, the limbic system, originally called the rhinencephalon or "smell brain", is considered the oldest part of the brain controlling emotion and mood fluctuations as well as memory and motivation - principal functions of the amygdala. Odorants can also elicit a variety of involuntary and largely unconscious physiological responses. Via the hypothalamus, the limbic system is also involved with visceral functioning including hunger, thirst, heart rate, blood pressure, breathing, sexual arousal, the sleep/wake cycle as well as hormonal secretions. The hippocampus, another primary limbic structure, is involved in the selection and transmission of information in working memory as well as short- and long-term memory transfer; the hippocampus also plays a role in various declarative memory functions (Hertz, 2009). Thus, the notion that aromas are emotionally evocative, influencing cognitive and underlying physiological processes is consistent with the neuroanatomy of the limbic system and its strong and direct connection with olfaction pathways. Consequently, it is not surprising that research suggests fragrances diffused into an environment influence fundamental psychological and physiological processes, affecting mood or emotional states, activation of memories, as well as arousal and cognitive performance. Theoretically, therefore, humans are unavoidably influenced to some extent by their olfactory environment; however, the science to support such assertions remains inconclusive. This thesis recognizes the shortcomings of previous studies in this 
field and relates it back to the present research in order to assert a valid and reliable paradigm.

\section{Effects of Lavender and Rosemary on Mood}

Although many argue that mood is greatly influenced by odour (Diego et al., 1998; Motomura, Sakurai, \& Yotsuya, 2001), no such evidence was found by Ludvigson and Rottman (1989) as self-report adjective checklists for mood yielded no detectable differences in the presence of the lavender aroma before and after a task involving arithmetic reasoning. Participants exposed to lavender did worse on the arithmetic task than those in the no odour condition; however, their poor performance did not influence their mood state as indicated by the adjective checklists. Interestingly, however, a separate index assessing participants' affective reaction to the experiment itself produced a contrasting result to the mood checklist. That is, participants exposed to lavender during the study reported a more positive affective reaction toward the arithmetic tasks, despite the aroma having a detrimental effect on their task performance. The authors propose that this finding suggests that the increased relaxation elicited by the lavender aroma could diminish cognitive functioning while at the same time elicit a favourable affective reaction (Ludvigson \& Rottman, 1989). It is conceivable that the subtle relaxing effect of lavender enhanced participants' mood favourably such that they reported enjoying a task they did poorly on but did not reach a threshold of awareness that would influence their responses on the mood questionnaire.

The influence of lavender on mood has also been extended to stress research. Lavender aroma has been shown to decrease the degree of perceived stress of a situation 
which may influence participants' mood regardless of task performance. For example, Motomura et al. (2001) effectively stressed participants by having them to sit alone with nothing to do in a soundproofed room and wait for experimental instruction for 20 minutes. After administering an anxiety checklist before and after the waiting experience, it was found that participants in this stressful condition yielded higher mean anxiety ratings than the control group who was not exposed to the soundproofed room or 20 minute time limitation; this indicated that the situation was in fact regarded as stressful to participants. With that said, those participants who were in the stressful condition and who were exposed to lavender during that period, perceived the situation as less stressful and reported a decreased negative mood than those receiving no aromatic exposure. Therefore, participants' feelings of stress increased in the psychologically stressful condition and this anxiety was reduced for those exposed to lavender aroma. Other researchers have further investigated the response to stress and other aspects of mood as moderated by fragrance inhalation.

In a study assessing the effects of rosemary, lavender, or water on mood states following an anxiety-provoking task (timed crossword puzzle), Burnett, Solterbeck, and Strapp (2004) conclude that the scents of rosemary and lavender have the potential to moderate various aspects of mood. It was found that rosemary exposure associated highly with scores on the tension- anxiety subscale of the Profile of Mood States (POMS) inventory. Participants in that condition reported feeling uneasy, tense, on edge, restless, and nervous. Furthermore, participants in the rosemary condition scored highly on confusion-bewilderment, indicating that they felt forgetful, unable to concentrate as well as feeling uncertain and inefficient. Considering that rosemary is 
believed to be a stimulating scent, the authors suggest that participants were likely overstimulated by the arousing scent in conjunction with the anxiety-eliciting crossword puzzle task. The researchers used commercially produced crossword puzzles rated as moderately difficult with solving times presented by publishers as requiring $30-45$ minutes; however, to increase anxiety, participants were asked to complete the puzzles in only 10 minutes. The findings of this study are in agreement with Moss et al. (2003) who suggested that rosemary inhalation can raise arousal levels to the extent that the individual becomes over-aroused, increasing the difficulty for performing stressful tasks.

Psychological Effects of Lavender and Rosemary on Cognitive Tasks

This section provides a critical review of studies which have investigated the effects of lavender and rosemary on various cognitive domains. Methodological inconsistencies will be discussed as they contribute to controversial findings regarding experimental outcomes. Possible explanations for conflicting evidence will be offered.

\section{Arithmetic Computation}

Previous literature on the effect of lavender on cognitive tasks offers contradictory evidence. For example, an early study (Ludvigson \& Rottman, 1989) dispersed a high concentration of a particular aroma continuously beginning 20 minutes before participants arrived, using two Aroma Disc Player diffusers, each diffusing $15 \mathrm{ml}$ of the essential oil with intensity set at maximum. A large exhaust fan was used to extract the room air for at least one hour between sessions in the within-subject study in which participants were exposed to a different scent in each session, including lavender as well as a control session with no scent. The 
researchers evaluated arithmetic reasoning by administering a task including 35 items where participants were presented with an arithmetic problem in words and had 13 seconds to select the appropriate arithmetic operation that would solve the problem from four choices offered. It was found that the high concentration of lavender adversely influenced participants' arithmetic functioning compared to the no-odour control condition.

In complete contrast to these findings and using imperceptible/unnoticed levels of scents, Degel and Köster (1999) demonstrated that participants made fewer errors in the presence of the lavender aroma than in the presence of no odour and statistically fewer than in the presence of the stimulant jasmine. Given that the present study also used a stimulating aroma (rosemary), it was assumed that the participants exposed to rosemary would perform worse than those exposed to lavender or no odour on arithmetic computations. The arithmetic test consisted of 5 blocks of 15 tasks. In each block there were 5 multiplication tasks (multiply single digit by a double digit number), five addition tasks (add a double digit number to a double digit number), and five subtraction tasks (subtract a double digit number from a double digit number). Participants were given at total of 8 minutes to complete all 15 blocks. Because the odour concentration was imperceptible, distraction or expectancy regarding the study was eliminated and the authors therefore conclude that the improvement was likely due to the effect of the lavender odour itself. Degel and Köster (1999) attempt to explain their results by alluding to the Yerkes-Dodson law, commenting that performing the tasks was likely stressful, and the sedative influence of lavender may have enhanced performance by reducing arousal levels. Had the participants not been stressed, the 
authors presuppose that this effect would not have been apparent.

The inconsistency of results observed among these studies may suggest that high concentrations of aromatic oil (as given in the Ludvigson and Rottman study) may be distracting or perceived as being unpleasant and, therefore, improvements on mathematical computations may be more likely if the aroma is not administered with such strong intensity. Another possible explanation for the discrepancies among the aforementioned studies relates to differences in the difficulty or perceived stressfulness of the computation tasks. While participants in the first study simply had to select a mathematical operation that would solve the problem, albeit under time constraint, participants in the latter study were required to actually compute the arithmetic problems. The difference in cognitive demand may have resulted in the latter study being perceived by the participants as stressful in nature. Therefore, while the first study suggests that the anxiolitic effects of lavender impair cognitive function, the latter study may indicate that the sedative-like aroma of lavender may in fact be beneficial if the participant is overstimulated by a task they perceive as stressful (Sakamoto, Minoura, Usui, Ishizuka, \& Kanba, 2005). This explanation would be consistent with the Yerkes-Dodson law which dictates that performance increases with cognitive arousal but only to a certain point. When levels of arousal become too high, performance will decrease (Yerkes-Dodson, 1908). The present research considered arithmetic reasoning in terms of actual mathematical computations as this approach, according to the literature, would most likely evoke a more aroused state in participants, rendering the effects of the aromas more evident and easier to measure. 


\section{Memory}

In a study assessing the olfactory impact of lavender and rosemary on cognitive performance, Moss and colleagues (2003) found significant effects of both aromas on memory. Participants $(n=144)$ were randomly assigned to one of three independent groups: lavender, rosemary, or control, and subsequently performed a cognitive task battery assessing a range of cognitive domains including target attention, memory, and vigilance. Four drops of the appropriate oil (or water for the control condition) was placed on a Tisserand Aroma-stream diffuser pad and placed under a bench in the testing room, with diffusion commencing 5 minutes prior to the testing of each participant. With respect to memory, the researchers found that participants exposed to rosemary outperformed those exposed to lavender on all memory task components. When examining performance on working memory specifically, it was found that both spatial and numeric working memory was significantly impaired for the lavender condition when compared to both the rosemary and control conditions, while both aromas slowed speed of memory performance relative to controls. Interestingly, participants in the rosemary condition reported feeling more alert than those in the lavender or control conditions, yet their performance was not faster in general. The researchers suggest this may be an indication that enhancements observed for memory may be independent of subjective state.

While Moss et al. (2003) found that memory is influenced by both rosemary and lavender, Ludvigson and Rottman (1989) reported that the ambient odour of lavender had absolutely no effect on memory (for a word recall task). Odour is a very 
encompassing context, one that cannot be ignored or avoided upon initial contact, albeit due to sensory adaptation, the experience of the odour diminishes considerably. When considering that memory is known to be highly contextually dependent and is easily disrupted by changes in contextual cues, the finding that memory recall was in no way influenced by odour stimuli was surprising and inconsistent with previous literature that demonstrates that contextual factors such as odours enhance recall (Davis, 1977; Herz, 1998). It is possible, however, that the contextual cue dissipates as sensory adaptation ensues. In fact, partial if not complete, adaptation can occur in less than a minute (Kaye, 2004). Despite being adapted to the odour and unaware of its presence at a conscious level, it is conceivable that the pervasive context remains regardless of conscious awareness. The authors concede that the absence of an effect on memory was unexpected, but provide no explanation regarding this finding. Perhaps the influence of lavender aroma on word recall is less robust than its influence on other memory tasks. The present study included memory tasks not limited to word recall alone, but also consisting of spatial and numeric working memory along with word and picture recognition tasks. Assessing the impact of aromas on various types of memory enabled the present research to be more specific in its conclusions regarding the effect of aromas on memory. Attention/Vigilance

Vigilance refers to “... a person's capacity of remain attentive to a task over a period of time (Van Toller \& Dodd, 1992, p. 58)." It is often measured by an individual's ability to detect unpredictable stimulus events over a prolonged duration. Vigilance tasks represent monotonous and understimulating situations wherein 
workload is very low. This understanding is the basis for the arousal model of vigilance, which holds that the repetitive and tedious nature of sustained attention tasks lowers the level of stimulation required by the central nervous system to maintain alertness. Understimulation may be caused by stimulus simplicity or repetition, effecting a reduction in the brain's responsiveness to external stimulation, ultimately producing a decrement in the efficiency of signal detection (Warm, Dember, \& Hancock, 1996). In laboratory settings, perhaps the most prominent vigilance effect is a decrease in signal detection after 20-30 minutes of continuous work (Jerison \& Pickett, 1963). Several studies have demonstrated that exposure to certain aromas augment signal detection despite following the inevitable pattern of vigilance decrement over time.

In the first study to demonstrate that olfactory stimulation can enhance the detection of visual stimuli, Warm et al. (1991) studied the effect of muguet (characterized as relaxing) and peppermint (characterized as stimulatory) on vigilance and subjective reports of stress and workload. Both aromas had high mean hedonic value as reported by participants. The authors hypothesized that the alerting aroma may reduce the stress of vigilance by enabling them to maintain the alertness necessary to successfully complete the task. Similarly, relaxing fragrances may also enhance concentration and performance on the task by reducing tension and feelings of stress resulting from performing the vigilance task. Therefore, authors reason that both stimulatory and relaxing fragrances may possess both performance and moodenhancing effects, yet for fairly different reasons.

In the vigilance task, participants were required to watch two parallel lines on a 
monitor. At random intervals, the distance between the lines would change at which point the participant was required to respond to the signal by pressing the spacebar on a keyboard. Aromas of muguet, peppermint, or no-odour were delivered to participants through oxygen masks for 30 seconds in every 5 minute period over the course of 40 minutes. As hypothesized, results indicated that both the aromas increased sensitivity to signals as compared to the control group, with false alarm rates equal in all groups. Aromas did not eliminate the effect of vigilance decrement insofar as all participants performed worse as the vigilance task progressed when compared to outset. Interestingly, neither of the aromas influenced subjective reports of stress and workload. This suggests that while both odours enhanced vigilance, participants were unaware of the effect the aroma had on their performance. This finding provides further support for the notion that subjective self-report data can insufficiently reflect bodily signals warranting more objective measures to be relied upon, as will be included in the present study. While Warm et al. (1991) found that stimulatory and relaxing aromas resulted in similar effects on vigilance performance, other more recent studies have found that only the sedating aroma of lavender proved beneficial in vigilance tasks when compared to alerting fragrances or no-odour conditions.

Shimizu et al. (2008) also investigated the effects of odours on sustained attention during a vigilance task. A number between 0 and 9 was shown every second on a computer screen and the participants were required to press a button only when they saw a " 0 " on the screen. Participants continued this task for a total of 30 minutes, with the first 2 minutes acting as a practice test. The stimuli consisted of lavender and eucalyptus essential oil compared to a control condition of no odour. The aroma delivery system 
included a complex series of instruments wherein the mixed gas passed through activated carbon for purification, then fed the bottle containing each odorant. The pressure on the bottle enabled the delivery of the odorant to the participant, with the gas being presented $10 \mathrm{~cm}$ below the nose. Participants received all conditions in random order within the 30 minute time frame for the task. It was found that, in general, incorrect responses as well as reaction time increased towards the end of the task; however, researchers found that lavender decreased reaction time and helped to maintain sustained attention during the 30 minute vigilance task when compared to controls. The authors suggest that the depressant activity of lavender on excessive arousal explains how a degree of attention was maintained throughout the task and a decrease of vigilance was inhibited even after a certain amount of time had elapsed. Generally, these findings seem counter to popular opinion that, in fact, stimulating odours are more effective for less challenging tasks such as this attentional task as they would keep individuals alert over time whereas sedating odours are more effective for more demanding tasks since excessive alertness causes vigilance to decrease. This notion was indeed substantiated by results from Moss et al. (2003) who found that performance for attention/vigilance tasks decreased significantly during exposure to lavender aroma when compared to controls. The administration of the sedating aroma of lavender likely further suppressed an already understimulated central nervous system. This finding suggests that lavender may decrease stimulation level (consistent with its reputed sedating properties), hampering performance on tasks that require high levels of mental alertness.

Although a decrease in signal detection occurs after 20-30 minutes of continuous work, the present research included a vigilance task component that took 
no longer than 10 minutes. This ensured that any decrease in vigilance could be attributed to the influence of the aroma rather than the decrease in signal detection that would be expected from prolonged continuous work at a sustained attention task. It is difficult to consider psychological effects of aromas alone as they act via the olfactory system and may thereby elicit physiological effects. Therefore, the following section pertains to the physiological effects of aroma inhalation.

\section{Physiological Effects of Lavender and Rosemary}

Researchers have implemented a wide variety of approaches to measure the effects of fragrances on humans including various physiological parameters. Many consider the role of olfaction to be of minimal significance as it occurs subconsciously; however, studies have demonstrated that olfactory stimuli can incite measurable physiological effects in humans. Inherently, physiological variables in humans are largely controlled by the autonomic nervous system. In general, the autonomic nervous system (ANS) functions are beyond conscious awareness, controlling visceral functions such as heart rate and respiration. Many studies that aim to measure changes in the ANS that are indicative of relaxation and alertness use a variety of physiological indices including electroencephalogram (EEG), blood flow (BF), skin conductance (SC), blood pressure (BP), and electrocardiogram (ECG) measurements. Although lavender has generally been shown to have a sedating-, and rosemary a stimulating effect, physiological indices remain inconsistent, meriting future study.

For instance, Diego et al. (1998) found ample evidence suggesting cerebral changes depending on fragrance exposure. The researchers provided either lavender 
or rosemary aromas to participants for three minutes and measured participants' EEG activity for three minutes before, during, and after the therapy sessions. A cognitive task of math computations was administered before and after the aroma session. Participants in both groups performed the computations faster after the aroma session; however, the lavender group demonstrated greater accuracy in their computations task than the rosemary group. The authors found that the lavender group showed increased beta power, which has been acknowledged as suggesting drowsiness. Conversely, the rosemary group showed decreased frontal alpha and beta power, suggesting increased alertness. The fact that both groups performed the task faster than the control group was surprising when considering that the lavender group did not demonstrate increased alertness in their EEG patterns as did the rosemary group. However, the authors concluded that, because the lavender group was more relaxed as indicated by their EEG recordings, they were therefore better able to concentrate on the cognitive task at hand, thereby enhancing accuracy.

While the above study measured differences in neurological response, other studies have attempted to assess changes in ANS functioning. For example, as previously discussed, participants in Motomura et al.'s (2001) mental stress study provided selfreport data indicating that lavender reduced the stressful experience of sitting alone for 20 minutes with nothing to do; however, no effects were found on the physiological measurements of mean heart rate, diastolic or systolic blood pressure taken before and after the treatment. In contrast, Saeki and Shiohara (2001) found various physiological changes in a study investigating the effects of inhaling the fragrances of lavender, rosemary, and citronella. Nine female participants experienced the following four tests in 
a random order: control conditions without fragrance, lavender, rosemary and citronella fragrances. The effects of citronella will be omitted from the following discussion. The effects of the treatments on the autonomic nervous system (ANS) were assessed using blood flow (BF), skin conductance (SC), blood pressure (BP) and electrocardiogram (ECG) measurements. For each subject, baseline parameters were established for $3 \mathrm{~min}$ prior to the studies and then measured for 10 min during the control or treatment sessions. Results of the physiological measurements indicate that lavender and rosemary elicited essentially contrary physiological reactions. While lavender produced an increase in $\mathrm{BF}$ and decreased $\mathrm{SC}$ and $\mathrm{BP}$, rosemary produced a decrease in $\mathrm{BF}$ and increase in $\mathrm{BP}$ immediately after inhalation, with no change in SC. None of the physiological parameters differed significantly in the control condition, indicating that the ANS changes that were elicited in the aroma conditions were caused by the inhalation of the fragrance itself. The authors conclude that lavender may, therefore, cause physical relaxation by inhibiting ANS activity. In contrast, rosemary appears to stimulate the ANS, causing feelings of arousal. Authors did not assess personal preference but acknowledge that this factor may also influence these effects on the ANS.

A critical analysis of the literature pertaining to the psychological and physiological effects of the inhalation of the essential oils of lavender and rosemary reflect mixed findings regarding the possible influence of these aromas on arousal and cognitive performance. It is difficult to compare results across studies due to methodological differences, including a lack of valid and reliable experimental protocols. To date, evidence surrounding the effects of scents on human cognition and physiology is overwhelmingly anecdotal, based on subjective self-report information. 
Even those studies that aim to establish objective assessments have produced equivocal results. Using a controlled environment, the current study, therefore, aimed to address this lingering controversy.

\section{Experimental Measures}

\section{Mood Questionnaire}

There are two popular mood questionnaires including the Profile of Mood States (POMS; McNair, Lorr, \& Droppleman, 1971) and the Positive Affect Negative Affect Schedule (PANAS; Watson, Clark \& Tellegen, 1988). The PANAS assesses how the participant has felt in 7 different time periods: (a) present moment; (b) today; (c) past few days; (d) past week; (e) past few weeks; (f) past year; (g) on the average (general). As such, the PANAS is a better measure of mood traits than mood states. Conversely, the POMS is designed to measure only how participants are feeling "right now". Because the present study was only interested in participants' mood at the moment they were filling out the questionnaire, the POMS was most suitable as it is designed to assess transient mood states and has demonstrated high internal consistency $(r=.95 ;$ McNair \& Lorr, 1964). Furthermore, several items on the POMS could also be used to measure subjective levels of arousal - a measure that was taken pre- and postexperimentally in the present research paradigm. Mood adjectives such as alert, energetic, fatigued, anxious, relaxed also provided indices of self-reported feelings of arousal; therefore, the POMS was used in the present experiment in order to best assess current mood states as well as subjective arousal levels.

Physiological Measurements

Although there are several physiological methods for assessing arousal in 
humans, the two most widely used measurements are skin conductance and heart rate (Hongratanaworakit, 2003). There are several advantages to the use of these two particular indices with respect to determining changes in autonomic nervous system activity. Skin conductance reflects one of the fastest responding measures of stress response and is one of the most non-invasive physiological measures of autonomic nervous system activity (Cacioppo \& Tassinary, 1990).

Heart rate is also a valuable indicator of an individual's autonomic nervous system activity and is widely used as an index for the measurement of the sedative and stimulatory effects of aromas. For example, Yamaguchi (1990) found that lemon aroma increased heart rate whereas rose aroma led to a decrease in heart rate. This finding was used to indicate that lemon aroma has a stimulating effect while rose possesses a sedative effect. Furthermore, Hongratanaworakit, Heuberger, and Buchbauer (2003) detected changes of heart rate in response to olfactory stimulation of sweet orange aroma. As was found for the lemon aroma, Hongratanaworakit et al. found that sweet orange aroma also caused significant increases in heart rate (and subjective alertness) after inhalation, indicating a stimulating effect of sweet orange aroma. Thus, it appears that the pattern of heart rate changes may provide scientific verification regarding the stimulating and sedative effects of aromas on human physiology. Therefore, measures of autonomic arousal used in the present study consisted of skin conductance and heart rate. In addition, cortical arousal was measured using electroencephalogram (EEG).

EEG measurements allow for an extreme level of sensitivity regarding cortical arousal. Brain waves can reflect human consciousness levels, psychological state, and 
degree of arousal. For example, in tense or stressful mental states, the beta wave is dominant and the alpha wave is inhibited, whereas in mentally relaxed states, the alpha wave is dominant. Aromas have been shown to produce cortical brain wave activity responses indicative of varying levels of arousal (Hongratanaworakit, 2004). Therefore, the present research utilized EEG in conjunction with measures of autonomic nervous system activity to assess arousal in response to olfactory stimulation.

\section{Arithmetic Computations}

The arithmetic test used here replicated the tasks in Degel and Köstster's (1999) study discussed earlier. The task consisted of five blocks of 15 mathematical computations. In each block, there were five multiplication tasks (multiply single digit by a double digit number), five addition tasks (add a double digit number to a double digit number), and five subtraction tasks (subtract a double digit number from a double digit number). Participants were given a total of eight minutes to complete all 15 blocks (75 computations). Experimental instruction stressed speed, urging participants to complete all computations as quickly and accurately as possible. The instruction was designed to elicit additional stress/anxiety in the participant, making the task itself more stressful and the effects easier to measure.

\section{Cognitive Drug Research (CDR) Battery}

Cognitive performance was assessed using the Cognitive Drug Research (CDR) computerized cognitive assessment battery which allowed for a wide array of established cognitive domains to be evaluated. The CDR, which has been used in over 1000 trials worldwide, is the most widely used automated cognitive test battery used in clinical trials 
and has been cited in over 250 publications and 456 published conference abstracts (United BioSource Corporation, 2009). The system itself assesses major aspects of cognitive function targeting attention and vigilance as well as short-term working memory and long-term episodic secondary memory. Furthermore, the CDR system includes a YES/NO response box that automatically records accuracy and time to the millisecond. This advantage removes experimental error that may occur when timing trials or calculating accuracy. Using a variety of substances, it has been shown that the CDR is reliable, valid, and sensitive to acute cognitive improvements (Moss et al., 1998; Scholey, Moss, Neave, \& Wesnes, 1999) and impairments (O'Neill, Hanks, White, Simpson, \& Wesnes, 1995; Ebert, Oertel, Wesnes, \& Kirch, 1998). For these reasons, the CDR task battery was employed in the present study. The following are the CDR tasks: Word presentation and immediate word recall. A series of 15 words was presented sequentially for 1 second, with a 1 second interval between words. The words were a mix of one, two, and three syllables. Immediately following word presentation, the computer display counted down 60 seconds, during which time the participant wrote down as many of the words they could recall from the list. Recall was scored for the number of correct words and incorrect words (not presented in the list).

Picture presentation. Twenty photographs were presented sequentially with a stimulus duration of 2 seconds and an inter-stimulus interval of 1 second.

Simple reaction time. The word YES appeared in the middle of the computer screen at varying intervals. Participants were asked to press the YES button as quickly as possible every time they saw the word YES. This task included 50 trials and the 
interval between trials varied randomly between 1 and 2.5 seconds. Reaction time was recorded in milliseconds.

Digit vigilance. A number was displayed constantly to the right of the computer screen. A series of 240 numbers was presented one at time in the centre of the screen at a rate of 80 digits per minute. 45 of these digits matched the constantly displayed digit. The participant was asked to press the YES button as quickly as possible whenever the digit in the centre matched the one on constant display. Response accuracy (\%), reaction time (ms), and number of false alarms were recorded.

Choice reaction time. Either the word YES or the word NO appeared in the middle of the screen at varying intervals. The participant was asked to press either the YES or NO button as quickly as possible to correspond with the word presented. The task included 50 trials (25 "YES" and 25 "NO"), and the interval between trials varied randomly between 1 and 2.5 seconds. Accuracy of response (\%) and reaction time (ms) were recorded.

Spatial working memory. A schematic image of a house was presented for 5 seconds. The house had nine windows displayed in a $3 \times 3$ pattern, four of which were illuminated and five were dark. A series of 36 presentations of the same house appeared but with only one window illuminated. The participant was asked to press the YES button if the illuminated window was one of the four lit in the original house or NO if it was not. Participants were asked to respond as quickly as possible. Sixteen of the stimuli required a YES response and 20 required a NO response. Accuracy and reaction time were recorded and a sensitivity index calculated. 
Numeric working memory. Five digits were presented one at a time for at a rate of one digit every second. After the digits were shown, the participants repeated them back to the experimenter to ensure they remembered them. If the participant could not remember the digits, they were redisplayed up to 2 times if required. A series of 30 digits was then presented and the participant pressed YES or NO as quickly as possible depending on whether the digit was believed to be one of the original 5 digits initially presented. Fifteen digits required a YES response and 15 a NO response. This task was repeated three times using different 5 digits each trial. Reaction time and a sensitivity index were calculated.

Delayed word recall. This task relates to the Word Presentation task. The computer counted down 60 seconds, during which time participants free-recalled as many of the words

from the list that they could still remember. They could write down the same words they wrote in the immediate word recall task, as well as any others they believed were on the list.

Word recognition. The 15 words initially presented in the word recall task were presented again in random order, interspersed with 15 new words. For each word presented, the participant pressed either YES or NO to indicate whether or not they recognized the word as being from the original list. Accuracy and reaction time were recorded and a sensitivity index was calculated.

Picture recognition. The 20 pictures previously presented were shown again in random order, interspersed with 20 new but similar pictures. The participant signified recognition by pressing the appropriate YES or NO button as quickly as possible. 
Reaction time and accuracy were recorded and a sensitivity index was calculated.

The CDR tasks were collapsed into six factors (4 global factors, 2 subfactors), derived from the battery by a factor analysis originally conducted and described by Wesnes, Ward, \& McGinty (2000) and used in previous studies (Wesnes et al., 1997, 2000; Kennedy al., 2000, 2001; Moss et al., 2003). The contribution of each individual task measure to each of the primary outcome factors is included in Figure 1. The factors and how they are determined is described below.

Primary Cognitive Outcome Measures

Quality of memory. Derived by combining the "Secondary Memory" and "Working Memory" factors (see below).

Secondary (episodic) memory subfactor. Derived by combining the percentage accuracy scores from all of the secondary memory tests: delayed word recall, delayed word recognition, immediate word recall, and delayed picture recognition. This factor reflects the ability to store, hold and retrieve information of an episodic nature (i.e. an event, a name, an object, a scene, an appointment etc.).

Working memory subfactor. Derived by combining the percentage accuracy scores from the two working memory tests: spatial memory task and numeric working memory.

Speed of memory. Derived by combining reaction times of the four computerized memory tasks: numeric working memory, spatial memory, delayed word recognition, and delayed picture recognition.

Speed of attention. Derived by combining the reaction times of the three attentional tasks: simple reaction time, choice reaction time, and digit vigilance. 
Accuracy of attention. Derived by calculating the combined percentage accuracy across the choice reaction time and digit vigilance tasks with adjustment for false alarms. 


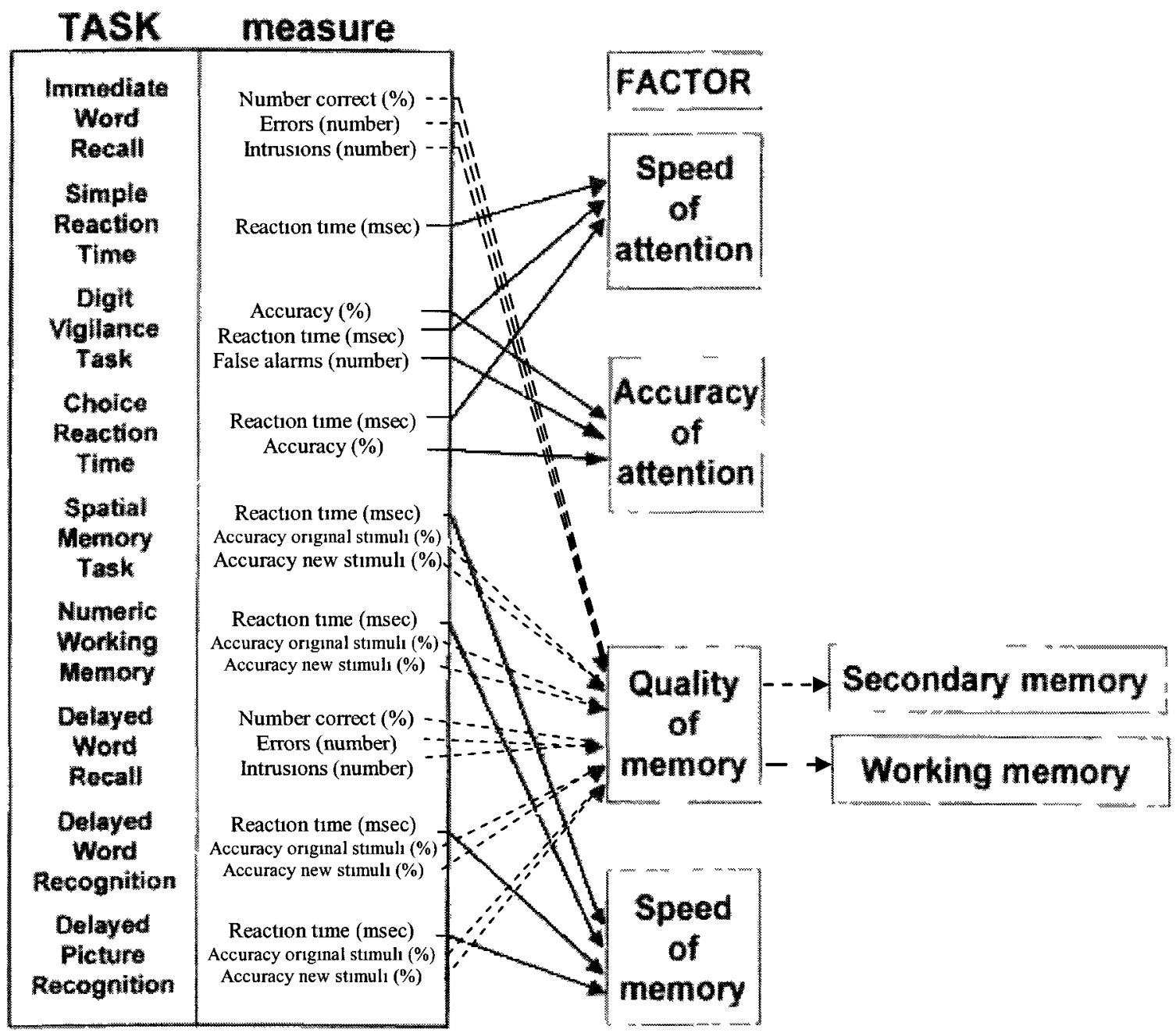

Figure 1. Schematic representation of the CDR battery showing all tasks, individual outcome measures, and the composition of the four factors derived by factor analysis. Arrows indicate that a task outcome measure contributes to the given factor of "Speed of Attention", "Accuracy of Attention", "Quality of Memory", or Speed of Memory". Dotted lines indicate the contribution to both "Quality of Memory" and to either "Working Memory" (- . -) or "Secondary Memory" (- - -), respectively (Moss et al., 2003). 


\section{Research Approach}

The primary objective of the present study was to determine the differential effects of the inhalation of the essential oils of lavender and rosemary on arousal and cognitive performance. This was accomplished by way of an experimental paradigm that assessed performance on a range of tasks relating to a number of established cognitive domains, and assessed arousal using sensitive electrophysiological technology equipped to measure autonomic as well as cerebral arousal levels. Participants were exposed to lavender, rosemary, or an unscented (control) environment and underwent the same methodological and procedural protocols such that any differences that emerged could be attributed to exposure to the scent itself. The cognitive tasks included some that were more difficult/stressful and others that were more simple/monotonous. If lavender is indeed sedating, then according to the Yerkes-Dodson theory of arousal, it should improve performance on the more cognitively demanding tasks, but impede performance on those tasks which are less demanding/monotonous. The opposite, therefore, should be true for rosemary. The following are specific hypotheses regarding the experimental outcome as derived from previous research and theoretical underpinnings:

$\mathrm{H} 1$ predicted that exposure to lavender would increase performance whereas exposure to rosemary would decrease performance on the working memory subfactor (including spatial working memory and numeric working memory) as well as on the secondary memory subfactor (including delayed word recognition, delayed picture recognition, immediate word recall, and delayed word recall) and speed of memory outcome measures of the CDR. The same was predicted to be true for the mathematical 
computation task, where lavender would increase performance and rosemary would decrease performance.

$\mathrm{H} 2$ predicted that exposure to rosemary would increase performance whereas exposure to lavender would decrease performance on the vigilance/attention tasks (simple reaction time, digit vigilance, and choice reaction time) that comprise the speed of attention and the accuracy of attention outcome measures of the CDR.

$\mathrm{H} 3$ predicted that participants in both scent conditions would give more positive mood ratings in the post experimental administration of the POMS scale than on the pre-experimental administration.

$\mathrm{H} 4$ predicted that participants exposed to rosemary would report an increase in arousal level whereas participants exposed to lavender would report a decrease in arousal post-experimentally compared with their pre-experimental measures.

$\mathrm{H} 5$ predicted that, in general, subjects exposed to rosemary would demonstrate an increase in ANS activity (i.e. heart rate and skin conductance measures would be higher), whereas subjects exposed to lavender would demonstrate suppressed ANS activity (i.e. heart rate and skin conductance measures would be lower), than control subjects.

H6 predicted that subjects exposed to rosemary would produce EEG patterns consistent with alertness (e.g., increased beta wave power), whereas subjects exposed to lavender would produce EEG patterns consistent with a more drowsy state (e.g., increased alpha wave power) compared to control subjects.

This study represented an important opportunity to advance the research into the potential for scents to manipulate humans on cognitive, physiological, and 
neurophysiolgical levels.

\section{Method}

\section{Participants}

A total of 90 undergraduate university students (males $=45$; females $=45$ ) were invited to participate in the study through Carleton University's online recruitment system (SONA). Participants were tested individually, and the entire experiment lasted approximately 60 minutes. In return for their participation, participants received one bonus percent toward their final grade for their Introductory Psychology course.

\section{Experimental Design}

The current study was a between-subjects experiment with participants assigned to one of three conditions: lavender, rosemary, or no-odour. The experimenter refrained from wearing any perfumes or deodorants on the days of testing to avoid contaminating the environment. To control for gender effects, each group was comprised of an equal number of males $(n=15)$ and females $(n=15)$ for a total of $n=$ 30 participants in each of the three conditions $(\mathrm{N}=90)$. In an unscented room (separate from the experimental room), participants first completed a mood/arousal questionnaire. Following that, they were brought into the experimental room and connected to the physiological equipment. Participants sat quietly for three minutes to establish a baseline measure on all physiological indices. Participants subsequently completed the CDR test battery as well as a paper and pencil arithmetic computation task. Tasks within the CDR were presented using the same presentation sequence for all participants. The arithmetic task was administered at the beginning for one half of the participants and at the end of the CDR battery for the other half. Following completion of the experimental tasks, participants completed the mood/arousal 
questionnaire for a second time. Equipment was then detached and participants completed a post-experimental questionnaire. Following this, participants were debriefed and asked to complete a document indicating their consent to use the data. The entire experimental procedure is shown in Figure 2 below.

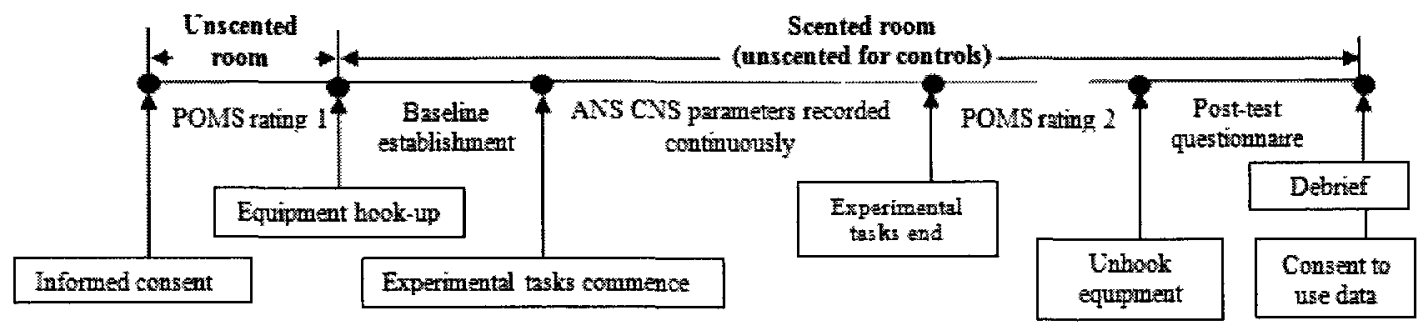

Figure 2. Diagram illustrating the experimental design.

\section{Materials}

Aromas. Natural essential oils of lavender and rosemary were purchased from OlfaCom, Incorporated. The essential oils come in the form of $60 \mathrm{~mm} \mathrm{X} 60 \mathrm{~mm} \mathrm{X} 47$ $\mathrm{mm}$ cartridges uniquely manufactured for use with the electronic diffusion system (OlfaCom Inc., A.C.2.i, Courbevoie, France).

Fragrance Delivery. This study attempted to ensure standardization of the experimental setting by way of the implementation of an electronic olfactory diffusion system (OlfaCom Inc., A.C.2.i, Courbevoie, France). The diffuser was activated three consecutive times prior to the participant's arrival into the experimental room, emitting 5 minutes of five bursts every seven seconds for each activation.

Cognitive Measures. Cognitive performance was assessed using the Cognitive Drug Research (CDR) computerized cognitive assessment battery which includes a range of measures that target attention, memory, and vigilance (please refer to pages 22- 
26 for a comprehensive section on the CDR task battery). A paper and pencil arithmetic task was added to the suite of cognitive tasks in order additionally test mathematical reasoning, a cognitive measure not included in the CDR task battery.

Mood Measure. The Profile of Mood States was used to assess participants' current mood. Participants rated 65 adjectives to indicate the extent to which each adjective described their current feelings on a 5-point adjective Likert rating scale (1 $=$ not at all to $5=$ extremely). From the 65 adjectives, six factors were derived: tension-anxiety, depression- dejection, anger-hostility, fatigue-inertia, vigour-activity, and confusion-bewilderment. Several items on the POMS could also be used to measure subjective levels of arousal; therefore, the present experiment analyzed the POMS for mood and analyzed a modified version of the POMS for subjective arousal.

Apparatus and Data Processing

A tailored version of the Cognitive Drug Research (CDR) computerized assessment system was employed to evaluate performance on a variety of cognitive tasks. The software was installed on a generic PC with an Intel Core 2 Quad processor, running Windows XP Pro. Stimuli were presented on a 24 inch widescreen LG LCD colour monitor, supporting a $75 \mathrm{~Hz}$ refresh rate and a 1920 x 1200 display resolution. Most tasks in the CDR required participants to respond via 'YES' and 'NO' buttons on a two-button response box, which recorded both accuracy and reaction time. Responses were made using participants' dominant hand.

Two autonomic nervous system (ANS) parameters and one central nervous system (CNS) measure were recorded simultaneously and in real time. The ANS 
parameters included skin conductance and heart rate; EEG was used to record brain waves. Skin conductance, heart rate and EEG were recorded with a ProComp Infinity system (Thought Technology, Montreal, Canada). All computerized biofeedback recordings were transmitted through a multi-modality encoder and connected directly to the $\mathrm{PC}$ in real time via a fiber-optic cable.

Skin Conductance (SC). SC amplitude in microsiemens $(\mu \mathrm{S})$ was recorded using the SC-Flex/Pro skin conductance sensor and two $\mathrm{Ag} / \mathrm{AgCl}$ finger electrodes (Thought Technology, SA9309M). The finger electrodes were placed on the first phalanx of the ring and the index fingers of the non-dominant hand and attached with Velcro.

Pulse Rate (PR). PR was recorded using the BVP-Flex/Pro sensor (Thought Technology, SA9308M). The photoplethysmographic probe was placed on the middle finger of the non-dominant hand and was used to assess heart rate in beats per minute (bpm).

Electroencephalography (EEG). Raw EEG signals measured in microvolts $(\mu \mathrm{V})$ was recorded using the EEG-Z sensor (Thought Technology, T9305Z) and was used to measure the quality of the signal. One EEG electrode was filled with EEG conductive paste. This electrode was placed on the midpoint of the participant's head (position $\mathrm{Cz}$ ). The other two electrodes clipped on to the participant's ears. For biofeedback purposes, real-time changes in the entire EEG bandwidth was measured to identify frequency subsets, where the most frequently observed subsets are defined as: 1-4 Hz (Delta); 5-7 Hz (Theta); 8-12 Hz (Alpha); 13-20 Hz (Low Beta) and 21-30 $\mathrm{Hz}$ (High Beta). 
Procedure

Upon arrival, participants were seated in an unscented room and the experimenter explained to the participant that the purpose of the study was to validate a new cognitive test battery as well as to assess any physiological changes to the person throughout the course of the test battery. The participant completed an informed consent form (see Appendix A), followed by a pre-experimental questionnaire (see Appendix B), and the POMS questionnaire (no appendix is provided for the POMS due to copywrite restrictions). Next, the participant was lead to the scented experimental room (or unscented room for the control condition) and connected to the skin conductance, heart rate, and EEG sensors. If the participant mentioned or acknowledged the scent in the room in any way, the experimenter dismissed the aroma by telling the participant that the experiment rooms are shared by many researchers doing a variety of different experiments. Before connecting each piece of equipment to the participant, the researcher first explained what kind of sensor she was going to attach to the participant as well as what body part it would be attached to. The area of skin under each sensor was first sterilized using rubbing alcohol. After the participant was fully connected to each device, (s)he sat quietly for three minutes, during which time baseline measures were being recorded for each physiological index.

Once baseline levels had been established, the experimenter reminded the participant that the purpose of the study was to test the efficacy of a cognitive task battery. For each task in the CDR, the instructions first appeared on the computer screen. Once the participant felt they understood the task requirements, they pressed the 
YES button to start the task (see Appendix C for CDR instructions provided by the United BioSource Corporation, 2009). The paper and pencil arithmetic task (see Appendix D) was counterbalanced such that it was administered before the CDR for one half of the participants and at the end of the CDR battery for the other half. The researcher explained the arithmetic task to the participant and justified its inclusion by telling the participant it would soon be part of the task battery they are testing. After completing all cognitive tasks, the participant again completed the same POMS questionnaire as they did pre-experimentally (however this time they were completing it in the scented environment). At this point, the researcher removed all electrophysiological equipment and administered some post-experimental questions (see Appendix E) which assessed the participant's rating of the pleasantness of the odour presented had they detected one (applicable only to those in the scented conditions) as well as various other questions of interest (applicable to all participants). Finally, participants were verbally debriefed regarding the nature of the experiment and were given a debriefing form to take with them (see Appendix F). Once the true nature of the study was known to the participant, (s)he was also asked to complete a form indicating that (s)he (still) permits the use of his/her data (see Appendix G).

\section{Results}

The outcomes of the cognitive performance tests are presented first - task-specific performance data will be followed by performance data on the primary outcome measures of the CDR. Next, results for the mood measures will be presented, followed by an analysis of the autonomic nervous system arousal measures. An analysis of the EEG data obtained will follow last. 
Cognitive Performance

This section on cognitive performance tests Hypotheses 1 and 2. First, Hypothesis 1 predicted that lavender would increase performance and rosemary would decrease performance on the following:

- working memory subfactor (including spatial working memory and numeric working memory)

- secondary memory subfactor (including delayed word recognition, delayed picture recognition, immediate word recall, and delayed word recall)

- speed of memory outcome measures of the CDR.

- arithmetic computation task

By contrast, Hypothesis 2 predicted that exposure to rosemary would increase performance whereas exposure to lavender would decrease performance on the vigilance/attention tasks (simple reaction time, digit vigilance, and choice reaction time) that comprise the speed of attention and the accuracy of attention outcome measures of the CDR.

To identify performance differences among the participant groups, a series of oneway ANOVAs were conducted for each task. Only those tasks that were yielded significant $\mathrm{F}$ tests are reported at length. ANOVA summary tables for these and all subsequent analyses are available in Appendix H. Response times are given in milliseconds and represent the mean speed of individual responses to all stimuli within the specific task.

Simple Reaction Time. Q-Q and box plots identified three extreme outlying cases which were omitted from subsequent analysis, and the Shapiro-Wilk test of normality 
indicated that normality could be assumed. The ANOVA yielded a significant main effect, $F(2,84)=5.26, p<.01, \eta_{p}{ }^{2}=.11$, observed power $=.82$. Means, standard deviations, and significant comparisons for this and all subsequent tasks within the CDR are presented in Appendix I. Homogeneity of variance was violated for this set of data, $F(2,84)=3.31, p=.04$, suggesting some heterogeneity of variance. Therefore, the Games-Howell correction for unequal variances was used for the post-hoc analyses. As shown in Figure 3 below, these indicated that participants in the lavender group took significantly longer to respond than both the rosemary and the control groups, $p<.05$ (see Figure 3) and that these two did not differ from each other.

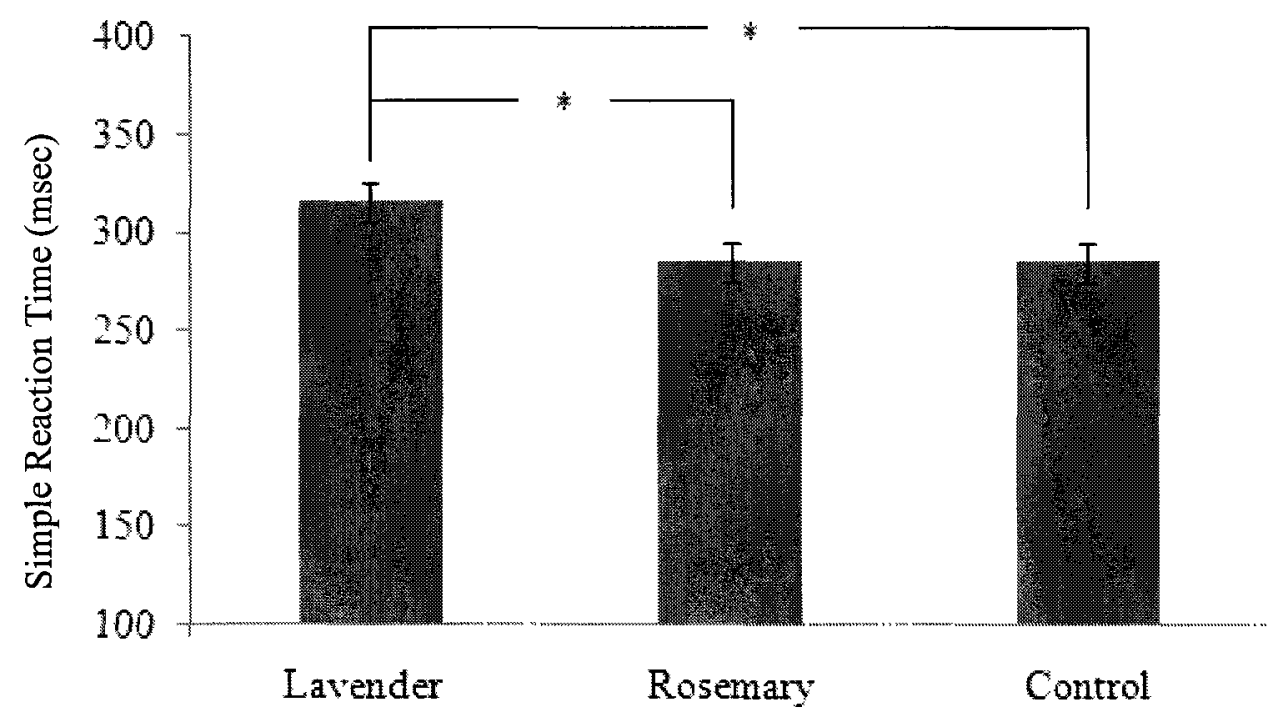

Figure 3. Effect of lavender and rosemary on the Simple Reaction Time task outcome measure of the CDR test battery. Figure depicts mean values. Error bars represent standard errors. ${ }^{*} p<.05$.

Digit Vigilance. One outlier was removed for the subsequent analysis as Q-Q and box plots identified it as an extreme value. Upon its omission, the assumption of 
normality was met. There was a significant main effect, $F(2,86)=5.47, p<.01, \eta_{p}{ }^{2}=$ .11 , observed power $=.84$, indicating an overall difference between the three participant groups. Levene's test indicated the existence of heterogeneity of variance, $F(2,86)=$ $3.51, p=.03$; therefore, the Games-Howell correction for unequal variances was used for the post-hoc analyses. These indicated that participants in the lavender group took significantly longer to respond than the control group, $p<.05$ (see Figure 4). No other significant comparisons emerged for response time. The finding that lavender did decrease performance on these vigilance/attention tasks provides support for Hypothesis 2. No significant difference was found for accuracy among the participant groups, $p>$ .05 .

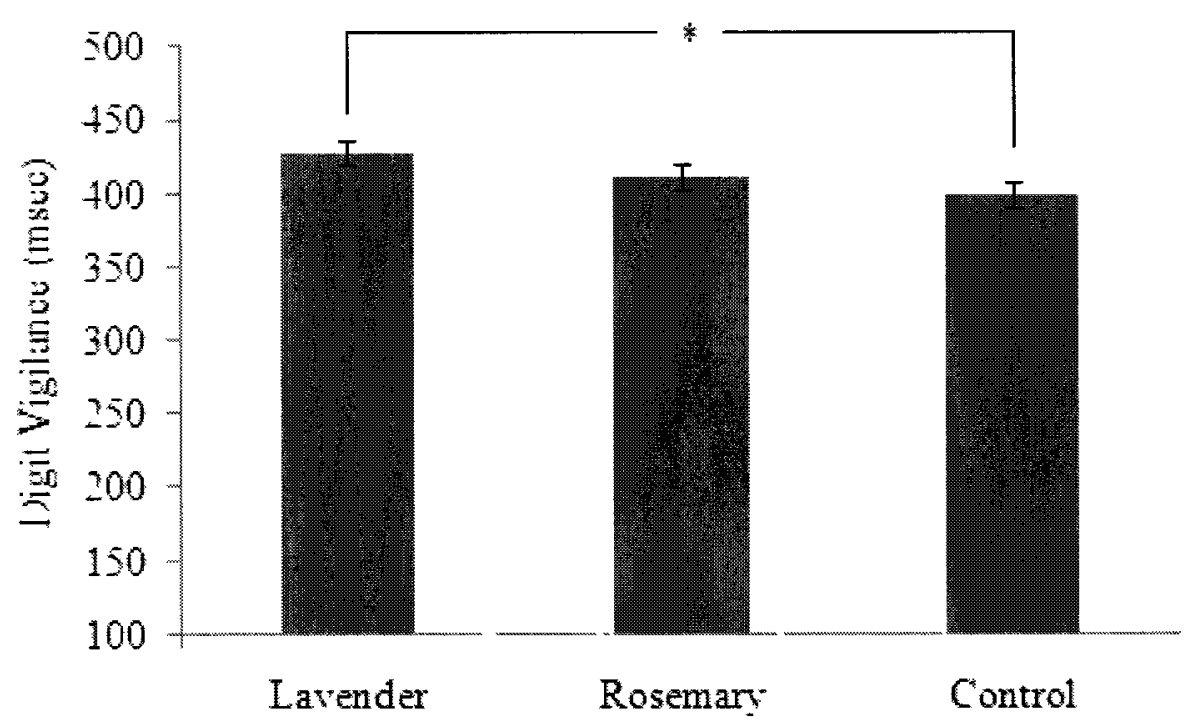

Figure 4. Effect of lavender and rosemary on the Digit Vigilance task outcome measure of the CDR test battery. Figure depicts mean values. Error bars represent standard errors. $* p<.05$. 
Spatial Working Memory. Q-Q and box plots identified six extreme outlying cases, several of who had been noted as having difficulty understanding the task. The time taken for the experimenter to clarify the nature of the task to these participants contributed to the prolonged response times. These cases were omitted from the subsequent analyses and the assumption of normality was met. A significant main effect for participant group was found, $F(2,81)=3.61, p<.05, \eta_{p}{ }^{2}=.08$, observed power $=$ .65 , indicating an overall difference between the three groups. Levene's test indicated the existence of heterogeneity of variance, $F(2,81)=7.57, p=.00$; therefore, the GamesHowell correction for unequal variances was used for the post-hoc analyses. These indicated that participants in the lavender group took significantly longer to complete the task than those in the control group, $p<.05$ (see Figure 5). No other significant effects were found. No significant difference was found for accuracy among the participant groups, $p>.05$. 


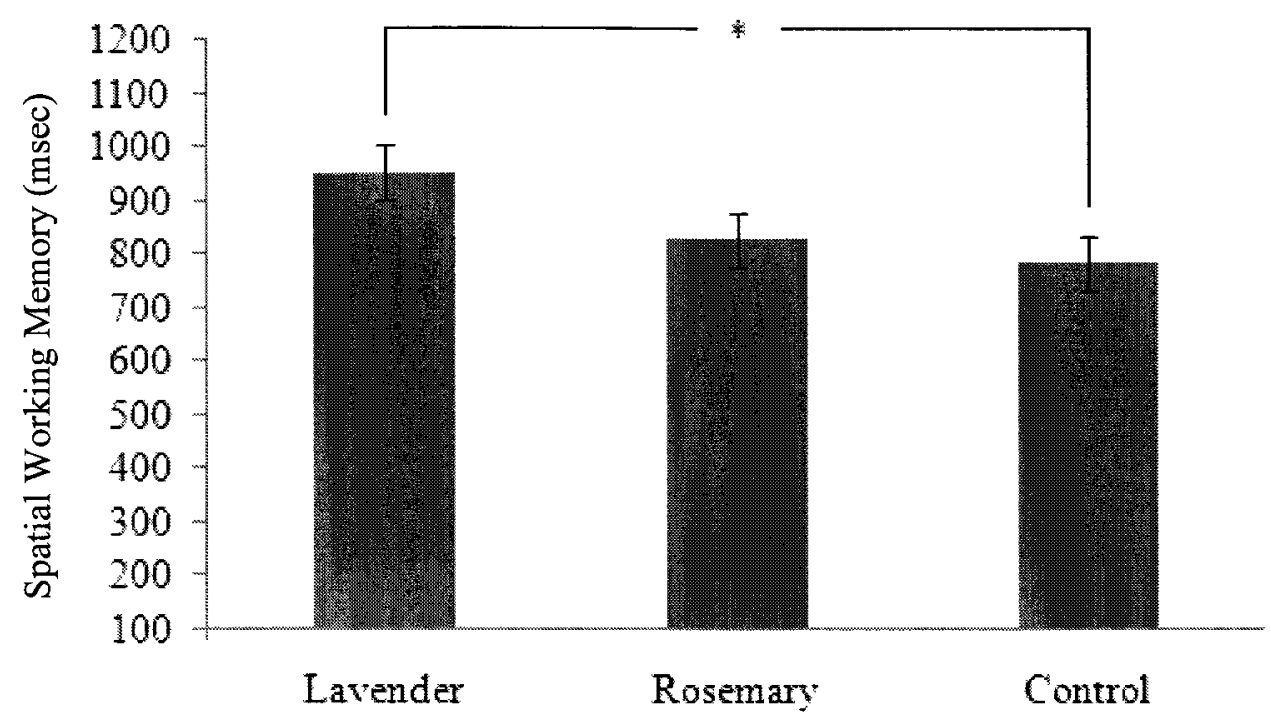

Figure 5. Effect of lavender and rosemary on the Spatial Working Memory task outcome measure of the CDR test battery. Figure depicts mean values. Error bars represent standard errors. ${ }^{*} p<.05$.

Contrary to the prediction of Hypothesis 1, no significant differences were found for immediate or delayed word recall, choice reaction time, arithmetic, word or picture recognition, or numeric working memory tasks, all $p>.05$. The following section concentrates on the analyses of the primary outcome measures.

\section{CDR Primary Outcome Measures}

Speed of Attention. A one-way ANOVA revealed a significant difference between participant groups, $F(2,84)=3.24, p<.05$, with homogeneity of variance assumed, $F(2$, $84)=2.09, p=.13, \eta_{p}{ }^{2}=.07$, observed power $=.60$. Post hoc comparisons using Tukey's HSD post hoc criterion indicated that the lavender group produced significantly slower responses than the control group, $p<.05$. This is shown in Figure 6 below. No other significant differences were found. 


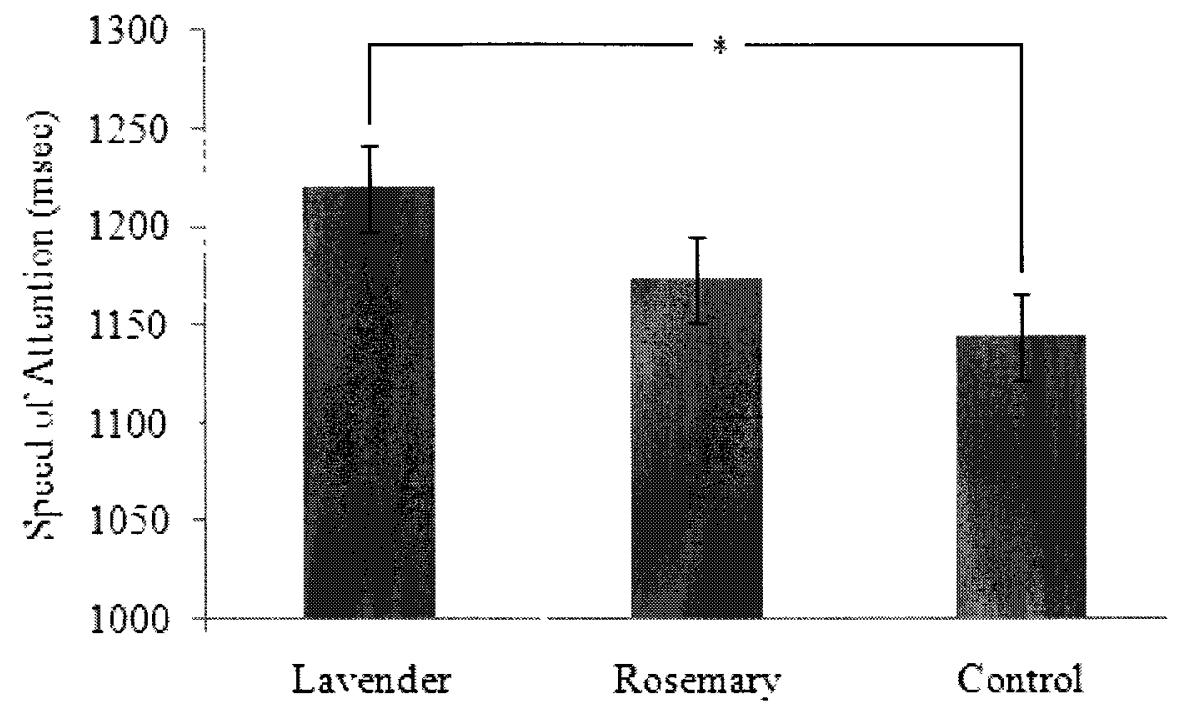

Figure 6. Effect of lavender and rosemary on the Speed of Attention factor derived from the CDR test battery. Figure depicts mean values. Error bars represent standard errors. $* p<.05$

Speed of Memory. This factor is the summation of four memory tasks, one of which was the spatial memory task. As mentioned above, the response time data for several participants was omitted for the spatial working memory task as the experimenter had to clarify the task to those participants. This increased participants' response times considerably and resulted in those cases presenting themselves as extreme outliers. In order still to include the data for those participants in the speed of memory analysis, the mean response time of the participants' other three memory tasks were used in the summation equation for the speed of memory factor. A one-way ANOVA revealed a significant difference between the participant groups, $F(2,87)=3.85, p<.05, \eta_{p}{ }^{2}=.08$, observed power $=.68$, with homogeneity of variance assumed, $F(2,87)=1.38, p=.26$. Post hoc comparisons using Tukey's HSD post hoc criterion indicated that the lavender 
group produced significantly slower responses than the control group, $p<.05$ (see Figure 7). No other significant differences were found.

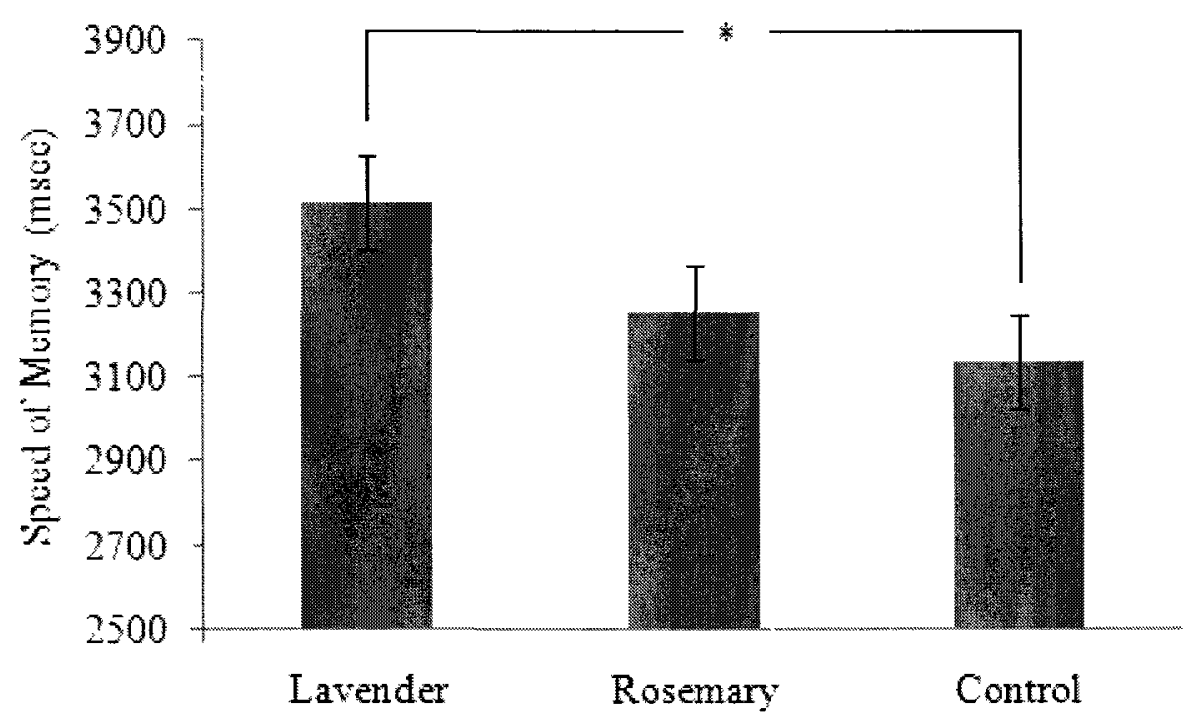

Figure 7. Effect of lavender and rosemary on the Speed of Memory factor derived from the CDR test battery. Figure depicts mean values. Error bars represent standard errors. $* p<.05$.

None of the one-way ANOVAs carried out for Accuracy of Attention, quality of working memory, quality of episodic memory, or arithmetic computation yielded a significant effect (all $p>.05$ ). Thus, scent did not affect performance on any of these tasks.

Taken together, these analyses indicate that exposure to a lavender-scented environment slowed down response times. This finding is consistent with the profile predicted for exposure to lavender, as would be expected with a decrease in arousal. Contrary to the prediction, however, the rosemary group did not respond faster than participants in the control group. No support was therefore provided for Hypothesis 1 
which predicted accuracy performance on memory tasks and on the arithmetic task would increase for the lavender group and decrease for the rosemary group, compared to controls. However, partial support was obtained for Hypothesis 2 which predicted that the lavender group would demonstrate decreased performance on vigilance/attention tasks.

\section{Mood Measures}

Normality of the Profile of Mood States (POMS) questionnaire data was assessed by comparing participant scores to the Adult Norms chart which accompanies the POMS questionnaire and is designed to be used to assess normality of scores. Post-experimental ratings of three participants in the lavender group were excluded from the analyses as they indicated that they had a very strong aversion to the scent of lavender and their mood ratings did not contribute to a normal sample. Hypothesis 3 predicted that participants in both scent groups would give more positive mood ratings in the postexperimental administration of the POMS than on the pre-experimental administration. Hypothesis 4 predicted that participants exposed to rosemary would report feeling an increase in arousal level (i.e., increase on the vigour-activity subscale and a decrease on the fatigue-inertia subscale) whereas participants exposed to lavender would report feeling a decrease in arousal (i.e., decrease on the vigour-activity subscale and increase on the fatigue-inertia subscale) post-experimentally compared with their pre-experimental measures. To test both these hypotheses, a (2) x 3 mixed randomized-repeated measures ANOVA was conducted for each of the six outcome factors of the POMS (tensionanxiety, fatigue-inertia, anger-hostility, confusion-bewilderment, depression-dejection, and vigour-activity). Administration time (pre-/post-experimental administration of the POMS) was the within-subject variable; group (lavender/rosemary/control) was the 
between-subjects variable, and the participants' score on each of the POMS factors was used as the dependent variable.

Main effects were found for four of the six factors. A main effect for tensionanxiety suggests that participants in general reported feeling more tense/anxious postexperimentally than pre-experimentally, $F(1,84)=11.41, p<.01$, with homogeneity of variance satisfied. A main effect of vigour-activity revealed that all participants reported higher vigour scores pre-experimentally than post-experimentally. This suggests that participants in all three groups experienced a decrease in feelings of vigour/activity at the end of the experiment. Furthermore, participants in general reported a decrease in their feelings of depression post-experimentally compared to their pre-experimental ratings, $F(1,84)=4.36, p<.05$, with homogeneity of variance met. Finally, a main effect of confusion-bewilderment revealed that participants in all groups reported feeling more confused at the end of the experiment than they did prior to its commencement, $F(1,84)$ $=7.58, p<.01$, with homogeneity of variance being met for this set of data. No main effects of participant group were found for any of the subscales. No difference was found for the Total Mood Disturbance (TMD) score, which is derived from scores on all six POMS subscales, indicating that, in general, participants' moods did not differ postexperimentally compared to pre-experimentally. These findings, therefore, refute Hypotheses 3 and 4 regarding mood and arousal. All descriptive statistics for the significant main effects are presented in Table 1. 
Table 1

Descriptive Statistics for POMS subscales yielding significant main effects.

\begin{tabular}{cccc}
\hline Scale & $\begin{array}{c}\text { Mean } \pm \text { SD } \\
\text { Pre-Experiment }\end{array}$ & $\begin{array}{c}\text { Mean } \pm \text { SD } \\
\text { Post-Experiment }\end{array}$ & Sample Size \\
\hline Tension-anxiety & $5.95 \pm 4.75$ & $7.93 \pm 5.52$ & 87 \\
Depression-dejection & $3.46 \pm 6.05$ & $2.48 \pm 3.95$ & 87 \\
$\begin{array}{c}\text { Vigour-activity } \\
\text { Confusion- } \\
\text { bewilderment }\end{array}$ & $14.45 \pm 4.98$ & $12.22 \pm 5.41$ & 87 \\
\hline
\end{tabular}

In sum, the POMS data provided no evidence to suggest that the experimental condition to which participants were exposed influenced their feelings of mood or arousal. In general, participants felt more tense/anxious and more confused/bewildered after than before participating in the experiment. Also, they reported a decrease in vigour/activity post-experimentally, suggesting that the experiment itself possibly had a fatiguing effect on them. Finally, their reported decrease in depression scores indicates that, although they felt more tense, confused, and fatigued, participants in all groups reported a more positive mood after participating in the experiment.

Autonomic Nervous System Parameters

Heart Rate. Statistical tests of normality were significant for all three groups (lavender, rosemary, control), indicating that they all differed significantly from a Gaussian distribution. Q-Q plots and box plots indentified three outliers, one in each participant group. After removing the three outlying data points, both KolmogorovSmirnov and Shapiro-Wilk statistical tests of normality became non-significant, indicating that none of the distributions differed significantly from a normal distribution. 
Using mean heart rate as the dependent variable, a one-way ANOVA was conducted on the three groups to test Hypothesis 5 predicting that autonomic nervous system activity, including heart rate, would be higher for participants exposed to rosemary and lower for participants exposed to lavender, than for participants in the control group. A significant main effect was found, $F(2,84)=5.87, p<.01, \eta_{p}{ }^{2}=.12$, observed power $=.86$.

Homogeneity of variance was violated for this set of data, $F(2,84)=3.79, p=.027$, indicating that heterogeneity of variance may be present. Therefore, although post hoc comparisons using Fisher's LSD tests indicated that the rosemary group experienced significantly higher heart rates than both the lavender and control group, upon correcting for the possible heterogeneous variance using Games-Howell post hoc criterion, heart rates were higher for participants in the rosemary group than for those in the lavender group, $p<.01$. This is shown in Figure 8 below. No other significant comparisons were found. The results therefore suggest that, collapsed across tasks, participants exposed to rosemary experienced an increase in heart rate activity compared to those exposed to the lavender. This finding offered partial support for Hypothesis 5 in that the individuals exposed to the rosemary-scented environment did experience higher heart rates than the lavender group, suggesting an increase in their autonomic nervous system activity. 


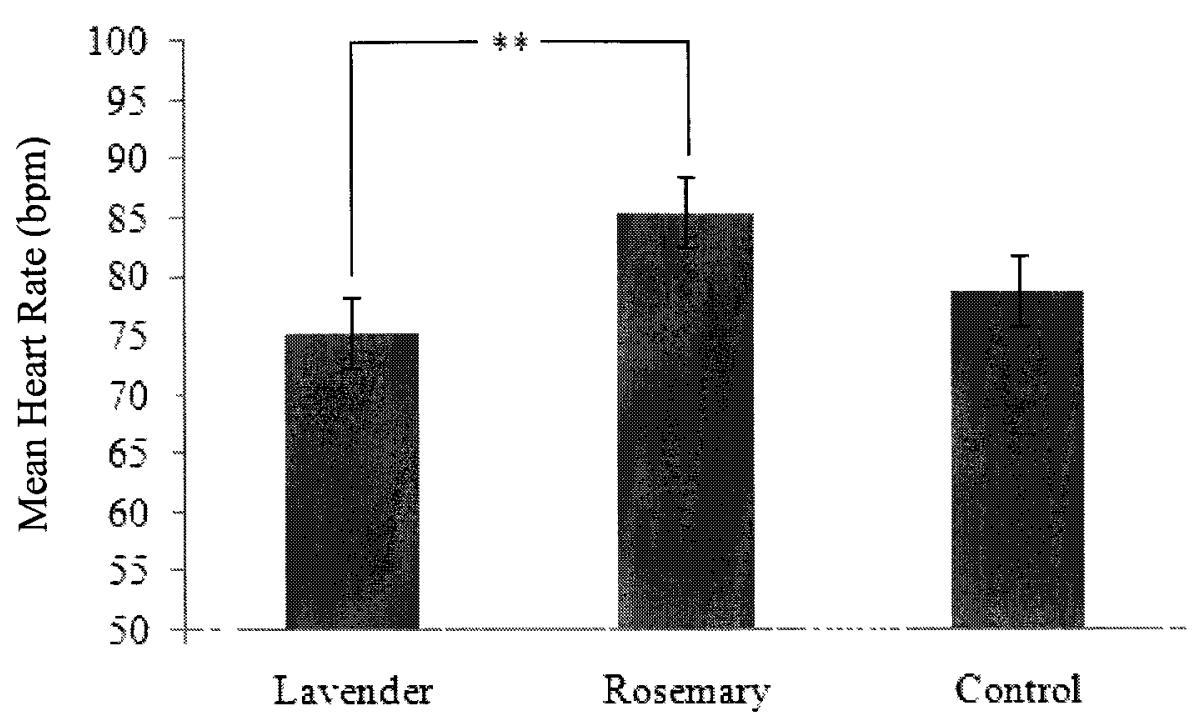

Figure 8 . Mean heart rate by participant group, collapsed across tasks. Error bars represent standard errors. ${ }^{* *} p<.01$.

A 3 x (13) mixed randomized-repeated ANOVA was conducted, using taskrelated heart rate as the dependent variable measured for the three groups for each task, including the baseline measure. Mauchly's test of sphericity was severely violated for this set of data, $p=.00$; therefore, the Huynh-Feldt correction was used for the subsequent analysis. A significant main effect of task-related heart rate was found, $F(4.52,379.68)=17.33, p<.001, \eta_{p}{ }^{2}=.17$, observed power $=1.00$. A main effect was also found for participant group, $F(2,84)=4.30, p<.05, \eta_{p}{ }^{2}=.09$, observed power $=.74$ (see Figure 9). 


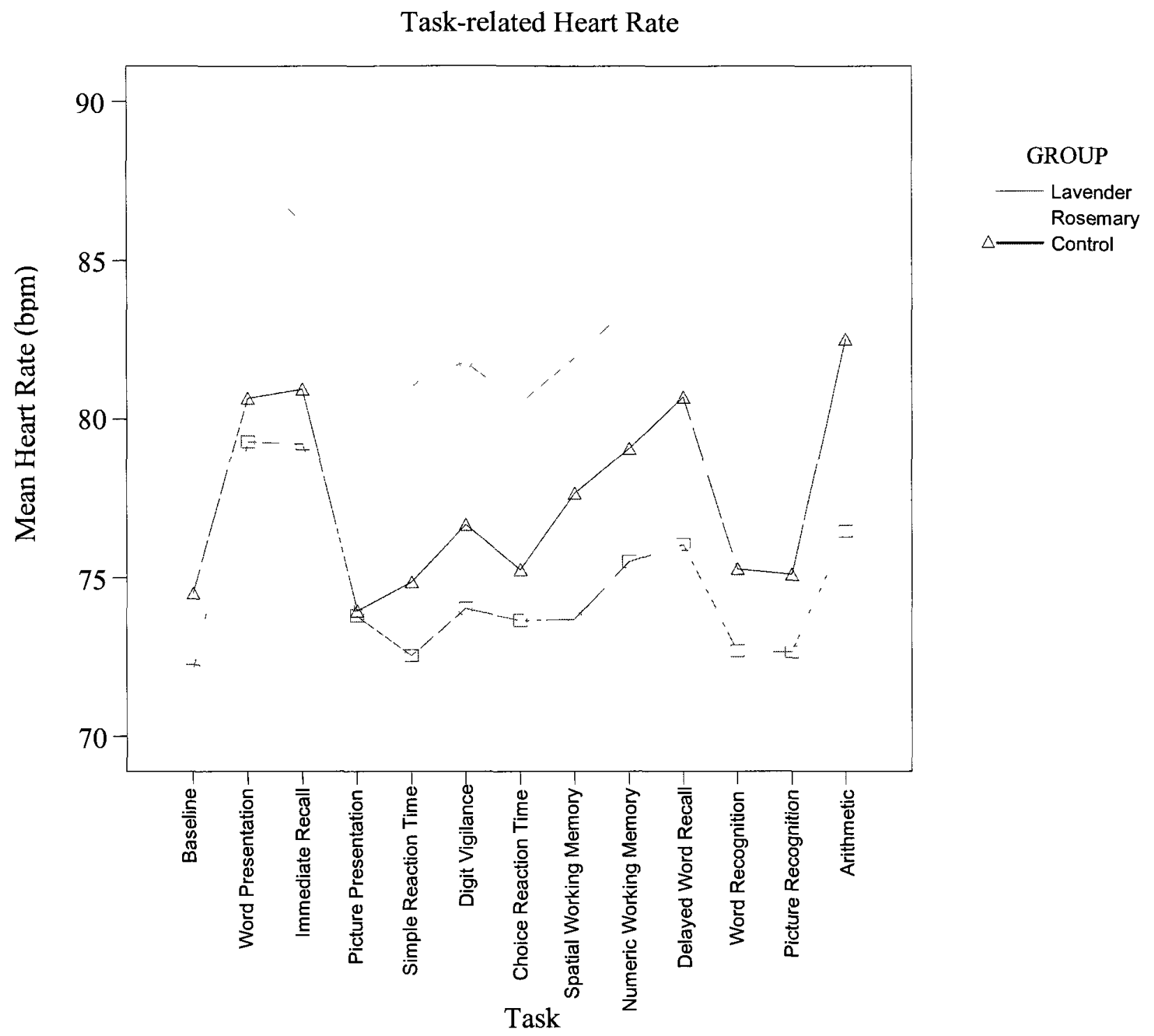

Figure 9. Task-related heart rate by participant group.

Inspection of Figure 9 shows a similar pattern for the three groups as they proceeded through the experiment, suggesting that the tasks had a similar physiological effect on each group, but that they varied only in relative magnitude: effect was somewhat attenuated for the lavender group and exacerbated for the rosemary group. 
A series of 12 one-way ANOVAs was conducted to identify the specific cognitive tasks that contributed to the significant variations in heart rate. Significant differences were found for seven of the 12 tasks, namely for simple reaction time $(F(2,84)=4.32, p$ $<.05, \eta_{p}{ }^{2}=.09$, observed power $\left.=.74\right)$; digit vigilance $\left(F(2,84)=3.43, p<.05, \eta_{p}{ }^{2}=\right.$ .08 , observed power $=.63)$; spatial working memory $\left(F(2,84)=3.33, p<.05, \eta_{p}{ }^{2}=.07\right.$, observed power $=.62)$; numeric working memory $\left(F(2,84)=3.27, p<.05, \eta_{p}{ }^{2}=.07\right.$, observed power $=.61)$; delayed recall $\left(F(2,84)=3.72, p<.05, \eta_{p}{ }^{2}=.08\right.$, observed power $=.67)$; word recognition $\left(F(2,84)=3.48, p<.05, \eta_{p}{ }^{2}=.08\right.$, observed power $\left.=.64\right)$; and arithmetic $\left(F(2,84)=5.65, p<.01, \eta_{p}{ }^{2}=.12\right.$, observed power $\left.=.85\right)$. No differences in heart rate were found for the remaining five cognitive tasks of word presentation, immediate recall, picture presentation, choice reaction time, or picture recognition, $p>$ .05. Post hoc tests using Bonferroni adjustment for multiple comparisons indicated that the significant differences found for task-related heart rate could be attributed to higher heart rates for participants in the rosemary group than for participants in the lavender group for all the significant $F$ tests (see Table 2). 


\section{Table 2}

For significant omnibus $F$ tests, mean differences for task-related heart rate by group as identified by Bonferroni multiple comparisons.

\begin{tabular}{|c|c|c|c|c|c|c|}
\hline \multirow{2}{*}{ Task } & \multirow{2}{*}{$\begin{array}{l}\text { Condition } \\
\text { (I) }\end{array}$} & \multirow{2}{*}{$\begin{array}{l}\text { Condition } \\
\text { (J) }\end{array}$} & \multirow{2}{*}{$\begin{array}{c}\text { Mean } \\
\text { Difference (I-J) }\end{array}$} & \multirow{2}{*}{$\mathrm{SE}$} & \multicolumn{2}{|c|}{ Confidence Intervals } \\
\hline & & & & & Lower & Upper \\
\hline \multirow{2}{*}{ Simple RT } & \multirow{2}{*}{ Rosemary } & Lavender & $8.70^{*}$ & 3.07 & 1.22 & 16.19 \\
\hline & & Control & 6.37 & 3.07 & -1.11 & 13.86 \\
\hline \multirow{2}{*}{ Digit Vigilance } & \multirow{2}{*}{ Rosemary } & Lavender & $7.75^{*}$ & 3.01 & .40 & 15.11 \\
\hline & & Control & 5.11 & 3.01 & -2.25 & 12.46 \\
\hline \multirow{2}{*}{$\begin{array}{l}\text { Spatial Working } \\
\text { Memory }\end{array}$} & \multirow{2}{*}{ Rosemary } & Lavender & $8.22 *$ & 3.19 & .44 & 16.01 \\
\hline & & Control & 4.26 & 3.19 & -3.53 & 12.04 \\
\hline \multirow{2}{*}{$\begin{array}{c}\text { Numeric Working } \\
\text { Memory }\end{array}$} & \multirow{2}{*}{ Rosemary } & Lavender & $8.01 *$ & 3.14 & .35 & 15.67 \\
\hline & & Control & 4.44 & 3.14 & -3.22 & 12.11 \\
\hline \multirow{2}{*}{ Delayed Recall } & \multirow{2}{*}{ Rosemary } & Lavender & $7.66^{*}$ & 2.83 & .75 & 14.58 \\
\hline & & Control & 3.02 & 2.83 & -3.90 & 9.93 \\
\hline \multirow{2}{*}{ Word Recognition } & \multirow{2}{*}{ Rosemary } & Lavender & $7.39 *$ & 2.84 & .45 & 14.33 \\
\hline & & Control & 4.79 & 2.84 & -2.15 & 11.73 \\
\hline \multirow{2}{*}{ Arithmetic } & \multirow{2}{*}{ Rosemary } & Lavender & $13.31 * *$ & 3.97 & 3.62 & 23.00 \\
\hline & & Control & 7.26 & 3.97 & -2.42 & 16.95 \\
\hline
\end{tabular}

${ }^{*} p<.05,{ }^{* *} p<.01$

To summarize, all three group displayed a similar pattern with respect to heart rates throughout the experiment. Differences were, however, found in the relative magnitude of the heart rates. That is, heart rate was somewhat attenuated for the lavender group, without reaching significance, and significantly exacerbated for the rosemary group relative to the lavender group. All differences in task-related heart rate were the result of the rosemary group producing significantly higher heart rates than the lavender group. These results are consistent with the profile predicted for exposure to rosemary wherein autonomic nervous system activity, including heart rate, was expected to be 
increased. However, no significant suppression of heart rate was found for the lavender group; therefore, these results provide only partial support for Hypothesis 5.

Skin Conductance. Two cases were omitted prior to any analysis as technical malfunctions with the skin conductance hardware produced obvious faulty recordings for these participants. Q-Q plots and box plots revealed two outliers in the skin conductance data - these points were removed for subsequent analyses. Both the KolmogorovSmirnov and Shapiro-Wilk statistical tests of normality were non-significant, indicating that the distributions were akin to a Gaussian distribution and, therefore, normality can be assumed. Collapsed across tasks, a one-way between-subject ANOVA revealed no significant differences for skin conductance among groups over the course of the experiment, $p>.05$. This finding provides no support for Hypothesis 5 predicting that autonomic nervous system activity, measured by both heart rate and skin conductance, would increase in the rosemary group and decrease in the lavender group, when compared to the control group. However, to determine whether differences exist for skin conductance levels for any specific task, an analysis of task-related skin conductance was conducted.

When examining skin conductance measures for each task, Q-Q plots and box plots revealed that participants 10 and 17 were creating outlying data points for several tasks. Participant 10 was an outlier in seven tasks and participant 17 in six tasks. The fact that these two participants presented extreme values in at least half of the tasks performed justifies their exclusion from subsequent analyses. A $3 \times$ (13) mixed randomized-repeated ANOVA was conducted, using task-related skin conductance as the dependent variable measured for the three groups for each task, including the baseline measure. Mauchley's 
test of Sphericity was violated, $p>.05$, therefore Huynh-Feldt's correction was used. A main effect of task-related skin conductance was found, $F(5.83,664.57)=50.21, p<$ $.001, \eta_{p}{ }^{2}=.39$, observed power $=1.00$, indicating that the type of task being performed influenced the participants' skin conductance levels (see Figure 10).

Task-Related Skin Conductance

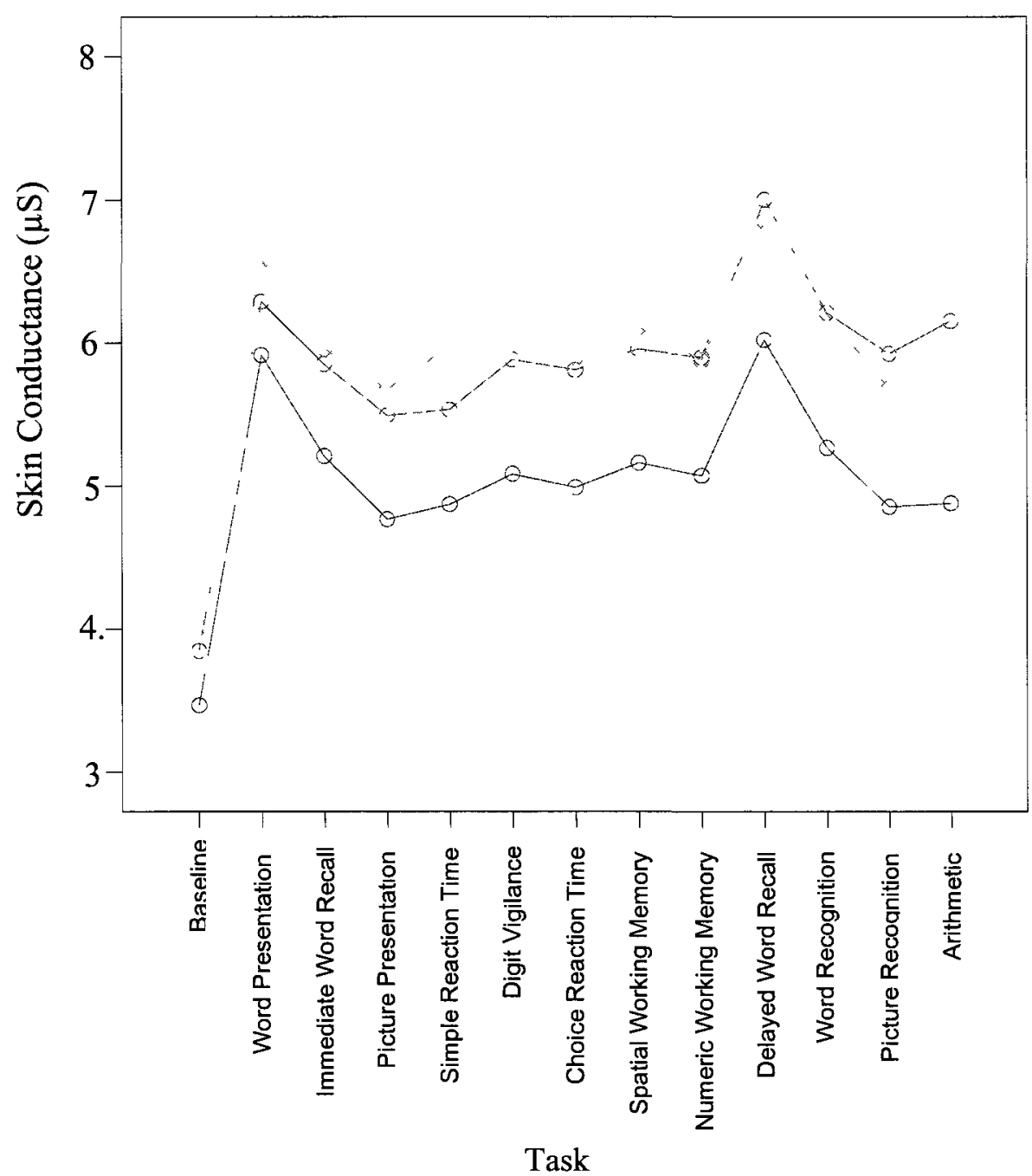

GROUP

Lavender

Rosemary

- Control

Figure 10. Task-related skin conductance levels by participant group. 
Visually, Figure 10 shows a similar pattern for the three groups as participants proceeded through the experiment, albeit with the control group experiencing somewhat lower levels of skin conductance than the scent groups at all times; however the lavender and control groups were indistinguishable from each other. Higher skin conductance levels are indicative of increases in autonomic nervous system arousal. Although individual tasks were analyzed with respect to skin conductance, they yielded nothing that could be meaningfully interpreted.

To summarize, the analysis of the skin conductance data did not provide support for Hypothesis 5 which predicted that, along with heart rate, skin conductance would also be increased for the rosemary group and decreased for the lavender group. Partial support was obtained, however, from the heart rate analysis which revealed that the rosemary group produced significantly higher heart rates than the lavender group, indicating increased autonomic nervous system arousal for this group. Electroencephalogram (EEG) Data

Technical malfunctions with the EEG hardware resulted in faulty recordings for four participants, which were therefore excluded from subsequent analyses. Alpha and beta baseline measures for case 26 and 43 were also excluded due to recording error; however, the error was rectified for the remainder of the experiment for these two cases.

It is difficult to define 'normal' alpha amplitudes as they vary greatly from person to person; however, a typical range is thought to be between 20 and 100 microvolts $(\mu \mathrm{V})$, with an average around $50 \mu \mathrm{V}$ (Ehlers \& Phillips, 2007). Beta waves have significantly lower amplitudes than alpha, with a typical range between 5-20 $\mu \mathrm{V}$, with an average usually around $5 \mu \mathrm{V}$ in normal adults; however, like alpha waves, beta waves also vary 
greatly between individuals. The Kolmogorov-Smirnov statistical test of normality indicated that the distributions for alpha and beta amplitudes for the three participant groups differed significantly from a normal distribution. However, because it is known that a high variability in the EEG bands of alpha and beta are typical from person to person, extreme individual differences were to be expected and, therefore, no specific cases were omitted from the analyses. ANOVA is robust to violations of normality with equal sample sizes and at least 20 degrees of freedom for error. These criteria were satisfied for these analyses and, therefore, robustness is expected.

To test Hypothesis 6 which predicted that that subjects exposed to rosemary would produce increased beta wave power, whereas subjects exposed to lavender would produce increased alpha wave power compared to control subjects, a one-way ANOVA was conducted for each frequency band, collapsed across tasks. Neither of these reached a level of significance, $p>.05$. Therefore, no support was found for Hypothesis 6 when comparing groups across tasks for EEG activity. However, it is possible that differences exist in EEG activity between the participant groups for specific task-related alpha and beta activity.

To determine whether differences in specific task-related alpha amplitudes exist between the groups, a series of one-way ANOVAs were conducted, one for each task as well as for the baseline. Homogeneity of variance was violated for each significant $F$ test; therefore, the Games-Howell correction for unequal variances was used for all post-hoc analyses. A significant difference was found for the baseline measure, $F(2,82)=3.58, p$ $<.05, \eta_{p}{ }^{2}=.08$, observed power $=.64$, suggesting that differences existed between the groups after being exposed to the experimental condition, but prior to the commencement 
of the cognitive task battery. Post hoc comparisons indicated that the lavender group had significantly lower levels of alpha amplitude power at baseline than both the rosemary and control group, $p<.05$. Means and standard deviations are presented in Table 3 below. A significant difference was also found for the picture recognition task, $F(2,83)=$ $3.58, p<.05, \eta_{p}{ }^{2}=.08$, observed power $=.64$, where the lavender group had significantly higher alpha amplitude levels than the rosemary group, $p<.05$. Finally, a significant difference was similarly found for the arithmetic task, $F(2,83)=3.35, p<.05, \eta_{p}{ }^{2}=.08$, observed power $=.62$, where the lavender group experienced significantly higher alpha amplitudes than the rosemary group, $p<.05$. No other significant differences were found for task-related alpha amplitude power. No significant differences were found for specific task-related beta amplitudes between the groups, $p>.05$.

Table 3

For significant omnibus $F$ tests, descriptive statistics and effects of alpha amplitude power by group, as identified by the Games-Howell post hoc criterion.

\begin{tabular}{ccccc} 
Task & $\begin{array}{c}\text { 1) Lavender } \\
\text { Mean } \pm \text { SD }\end{array}$ & $\begin{array}{c}\text { 2) Rosemary } \\
\text { Mean } \pm \text { SD }\end{array}$ & $\begin{array}{c}\text { 3) Control } \\
\text { Mean } \pm \text { SD }\end{array}$ & $\begin{array}{c}\text { Significant } \\
\text { comparisons }\end{array}$ \\
\hline Baseline & $6.20 \pm 2.71$ & $11.37 \pm 10.30$ & $9.91 \pm 7.14$ & $\begin{array}{c}1 \text { vs } 2^{*} \\
1 \text { vs } 3^{*}\end{array}$ \\
$\begin{array}{c}\text { Picture } \\
\text { Recognition }\end{array}$ & $8.90 \pm 6.70$ & $5.50 \pm 2.58$ & $6.83 \pm 4.22$ & 1 vs $2^{*}$ \\
Arithmetic Task & $14.58 \pm 16.25$ & $7.01 \pm 5.61$ & $9.62 \pm 8.91$ & 1 vs $2^{*}$ \\
\hline$* p<.05$. & & & &
\end{tabular}

Interestingly, there was a trend for the control group towards higher alpha and beta amplitudes than the scent groups, but the difference did not reach significance (see 
Figure 11 below). Further, an independent samples t-test revealed that, collapsed across group, there was no significant difference between the alpha $(M=10.61, S D=10.09)$ and beta $(M=13.14, S D=18.18)$ amplitudes, $p>.05$. This is very surprising considering that alpha amplitudes are generally much larger (normal adults $=50 \mu \mathrm{v}$ ) than beta amplitudes (normal adults $=5 \mu \mathrm{v}$ ). This finding may therefore suggest that alpha activity was attenuated or inhibited throughout the experiment, probably due to relatively high arousal levels. That is, participants were most likely attentive, anxious, or stressed, or, alternatively, in a state of expectancy to external stimuli.

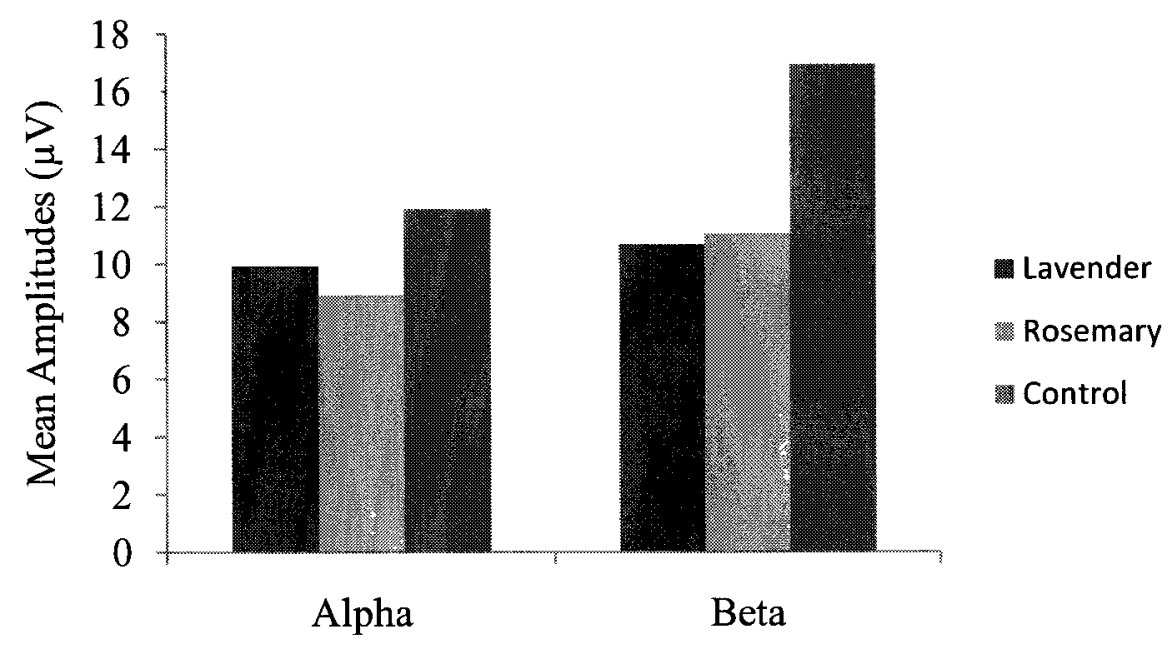

Figure 11. A comparison of mean alpha and beta amplitudes by participant group, collapsed across tasks.

Hypothesis 6 received very little support from these analyses. That said, however, at rest (baseline), the lavender group did generate stronger alpha amplitudes than the other two groups. Further, during certain times within the experiment, the lavender group did produce increased alpha power compared to the rosemary group. Therefore, some support was provided for the prediction that lavender exposure would relate to an 
increase in alpha. No support was obtained from the analysis of the beta waves. It is of interest to stress the extremely low alpha amplitudes and the larger than normal beta amplitudes produced by all participants in general. This pattern strongly suggests that all participants, regardless of the condition they were exposed to, experienced inhibited or blocked alpha waves, likely dominated by the beta frequency band which suggests that the experiment in general aroused the participants' central nervous system well beyond the adult average norm.

\section{Discussion}

This section summarizes the main findings of the formal experiment in this thesis and presents the contributions these findings make in advancing the research into the 7 potential for scents to manipulate humans on cognitive, physiological, and neurophysiological levels. This is followed by an outline of the limitations of the experiment. The final section describes future research.

\section{Summary of the Main Findings and Contributions}

The goal of this thesis was to determine the differential effects of the inhalation of the essential oils of lavender and rosemary on arousal and cognitive performance. This was done by exposing individuals to either a lavender-scented environment, a rosemaryscented environment, or a control (unscented) environment and measuring their cognitive performance as well as their subjective feelings of mood and arousal, and obtaining their physiological indices of arousal.

The results demonstrate that the inhalation of ambient aromas of essential oils can significantly affect aspects of cognitive performance and physiology. Due to the fact that only five out of the 60 participants who were assigned to scent conditions reported 
noticing a scent at all, these effects are considered to be valid irrespective of participants' expectations or beliefs. Lavender essential oil is generally thought to possess sedating properties and rosemary is believed to be stimulating - these well-known characteristics were reflected in the effects on cognitive performance and physiological reactions observed here.

Contrary to predictions, however, the exposure to lavender did not increase performance on the working memory subfactor, the secondary memory subfactor, or the speed of memory outcome measure of the CDR, nor did exposure to rosemary increase performance on the accuracy of attention outcome measure of the CDR. Consideration of the mean levels of accuracy in the tasks that combine to create these factors suggest that performance was effectively equal across conditions and that ceiling levels were being achieved for several outcome measures. For example, accuracy levels higher than $90 \%$ on tasks comprising the working memory subfactor as well as the accuracy of attention outcome measure indicate that a very minimal amount of enhancement could have been achieved and, the nature of the tasks within the CDR is such that subtle impairments do not produce detectable decrements in accuracy of performance compared to controls (Simpson, Surmon, Wesnes, \& Wilcock, 1991). Therefore, it is perhaps not surprising that no effect of participant group was observed in this experiment for accuracy measures.

Effects, however, were found for measures of reaction time for several tasks and outcome factors. With respect to the speed of memory factor, the lavender group displayed significantly longer reaction times compared to controls, but not to the rosemary group. This same pattern was observed for reaction times associated with the 
speed of attention outcome measure, where the lavender group produced longer reaction times compared to controls, but not to the rosemary group. These findings are consistent with the profile predicted for lavender, as would be expected from the reputed sedating properties of lavender. Further, when considering the relationship between arousal and performance as described by the Yerkes-Dodson theory of arousal, it is possible that the lavender group was functioning at a lower-than-optimal level of arousal, resulting in a decrement in reaction time performance compared to controls. Certainly the physiological heart rate data obtained for the lavender group provides support for this notion of the lavender group experiencing a suboptimal level of arousal as the participants exposed to lavender did produce significantly lower heart rates for the duration of the experiment compared to the rosemary group and somewhat lower than the control group, yet without reaching the level of significance set for the analysis.

It is surprising however, that reaction time was not enhanced for the rosemary group as a consequence of the stimulating properties associated with rosemary. That being said, it is quite possible that both the control and rosemary groups were functioning at an optimal level and the tasks themselves were so simple that further enhancement was not possible.

The physiological data obtained for the rosemary group provides a great deal of support for the notion of rosemary being a stimulant by nature. Averaged across the experiment, participants in the rosemary group produced an average heart rate of $85 \mathrm{bpm}$ - a full $10 \mathrm{bpm}$ faster than the average heart rate produced from lavender group participants and six bpm faster than those in the control group. The physiological heart rate data obtained for the rosemary group indicated that this group experienced 
significantly elevated heart rates compared to the lavender group for the two outcome factors with which a speed component is associated: speed of attention and speed of memory. Specifically, the rosemary group had higher heart rates for two out of the three tasks that constitute the speed of attention outcome measure (simple reaction time and digit vigilance) and for three out of four of the tasks that comprise the speed of memory outcome measure (spatial working memory, numeric working memory, and delayed word recognition), when compared to the lavender group. This suggests that the rosemary group was experiencing increased physiological arousal levels; however, their increased autonomic nervous system activity did not benefit them for this selection of tasks. Therefore, an increase in physiological arousal was not associated with a marked improvement in cognitive performance; however, a decrease in arousal was strongly associated with a decrement in performance. A possible explanation for this is that the cognitive battery itself was too simple such that, as with the accuracy levels, there was little room for improvement in reaction time if operating at optimal arousal levels; yet, if operating at a suboptimal level of arousal, as was the case with the lavender group, then impairments with respect to reaction time appears to be the consequence.

Heart rate is a very sensitive measure for variations in autonomic nervous system activity and is widely used as an index for the measurement of the sedative and stimulatory effects of aromas and, no doubt, the heart rate data obtained for this experiment was a good indicator of the participants' objective arousal levels. However, the same was not true for the skin conductance measurements in this study, where no variations were found between groups. The reliability of skin conductance has been reported as questionable in the literature, such that skin thickness can greatly influence 
skin conductance measurements (Revelle \& Loftus, 1992). Further, it was noted during pilot testing that pinching the metal on the probes harder, resulted in lower resistance (higher conductance). These factors may have contributed to the lack of support for the prediction that skin conductance measures would, as in the case for heart rate, be indicative of an increase in arousal for the rosemary group and a decrease in arousal for the lavender group. As a result, the heart rate measure of autonomic nervous system activity may, for the purpose of this study, be a more robust indicator of physiological arousal.

Despite an increase in physiological arousal during the study, as indicated by elevated heart rate, the rosemary group did not report feeling more aroused postexperimentally than the other groups. This suggests that participants' self-reported reactions to the fragrance inhalation may not reflect the individual's objective physiological reaction to the scent. Caution should be exercised when interpreting previous research that has relied solely on participant's self-report feelings of arousal to determine the effect the scent is having on the individual. Participants in all groups reported an increase on the vigour-activity subscale of the POMS, yet interestingly they did not report a corresponding decrease on the fatigue-inertia subscale. However, the creators of the questionnaire state that the two do not represent opposite indices (McNair et al., 1992). In fact, McNair and colleagues (1992) state that the vigour-activity subscale is associated with positive affect, where high scores indicate that the participants feel carefree and cheerful. Therefore, without a decrease on the fatigue-inertia subscale, increased scores on the vigour-activity subscale alone are not indicative of increased feelings of arousal. High scores reported post-experimentally for confusion-bewilderment 
may have been the result of completing such a wide variety of tasks in a relatively short amount of time. Alternatively, the requirement to complete the same questionnaire twice may also have added to their confusion.

With reference to the EEG data, no differences were found for either the alpha or beta frequency bands between groups, across tasks. This was unexpected when considering the reputation for lavender as being relaxing (indicated by higher alpha amplitudes) and rosemary as being arousing (indicated by higher beta amplitudes). However, prior to the commencement of the task battery, those exposed to lavender did produce significantly higher alpha power than both the rosemary and the control group. Therefore, a possible explanation for this lack of effect between groups during the experiment itself may be due to the nature of the task battery. That is, the tasks themselves required participants to be attentive, vigilant, work as quickly as possible, maintain information in their working memory, etc. Although the tasks themselves may not have been particularly challenging (evidenced by the ceiling effects on several of the tasks), the battery itself required the participants to maintain a certain level of engagement, and the continuous nature of the battery likely left the participants in a constant state of expectancy. The finding that, collapsed across group, no difference existed between the alpha and beta amplitudes was very interesting and unexpected. The reason being that alpha amplitudes are generally much larger (normal adults $=50 \mu \mathrm{V}$ ) than beta amplitudes (normal adults $=5 \mu \mathrm{V}$ ). The fact that all participants, regardless of condition, experienced such an inhibition of alpha power to the point where alpha and beta power were indistinguishable does support the notion that the task battery was sufficiently stressful or, at the very least, cognitively arousing. Therefore, despite the 
literature suggesting that lavender would cause participants to be more relaxed and therefore have higher alpha amplitudes, this pattern could not manifest likely because the circumstances would not allow these participants to experience the calming or relaxing effect that lavender is purported to have on the central nervous system. Had the tasks been more mundane, perhaps then the effect of lavender would have had a chance to emerge on a more global level. This also provides an explanation for the lack of support for the prediction that those in the rosemary group would produce an increase in beta power relative to controls. That is, the task battery itself was sufficiently cognitively engaging that it mentally aroused all participants regardless of the condition to which they were exposed. Again, had the task battery been more tedious or at least less cognitively stimulating, perhaps the pattern of increased beta activity for the rosemary group would have manifested when compared to controls.

The results of this study demonstrate that the inhalation of ambient aromas of essential oils can alter heart rate activity and aspects of cognitive performance. The physiological effects were not mirrored in self-report data regarding mood state which calls into question the validity of relying simply on participants' subjective feelings of arousal, as commonly practiced in olfactory research. The current findings provide mixed support for the notion of clear and opposing cognitive and physiological profiles for the aromas of lavender and rosemary; however, their respective sedating and stimulating characteristics were reflected in the cognitive and physiological data, providing some empirical substantiation for the reputed effects of lavender and rosemary. 


\section{Limitations}

Although the diffuser used to scent the experimental room was used the same for each of the scent conditions, there is no guarantee that the level of concentration was identical for both scents. Also, very few participants reported noticing a scent; therefore, although concentration levels were perceptible, perhaps stronger effects would have been observed had concentration levels been higher. Higher concentrations that would result in all participants perceiving the scents would have been beneficial to be able to investigate the extent that perceived pleasantness of the scent would have on the effects elicited.

A further limitation to the study was the baseline measurement. It was not possible to hook the participants up to all of the physiological equipment in a control room, record baseline there, then transport them with all the equipment still hooked up to them into the experimental scented room. As a result, baseline measurements had to be taken while the participant was in the scented room, but before they started the task battery. Therefore, it was not a true baseline. However, taking measurements during this period of time was a good indicator of the effect that the scent had on the participants when they were at rest. Given the circumstances, taking true baseline measures was not possible and therefore no data were available to assure that no differences existed between the three groups prior to the onset of the experiment. Certainly there is no reason to believe that the groups would differ in any way; however, without baseline measures, this claim cannot be confirmed.

\section{Future Work}

This thesis along with previous research suggests that the inhalation of ambient aromas can affect some aspects of cognitive performance and autonomic nervous system 
activity. Research in this field would benefit from the development of a very specific type of cognitive task battery that would be sensitive to more subtle changes in the cognitive performance of normal adults resulting from exposure to ambient aromas. Such a task battery could therefore offer a more nuanced understanding of the enhancement or impairments incurred from the exposure itself, avoiding the possibility of ceiling effects masking the outcomes.

Future research into the underlying mechanisms of action for lavender and rosemary aromas is necessary to better understand the effects of these scents on physiological reactions and cognition. Elucidation of the pharmacological mechanisms responsible for changes in the autonomic nervous system/central nervous system/or endocrine systems is crucial to our understanding of the influence that scents can exude on human physiology and behaviour. For a volatile compound to act pharmacologically, it must enter the bloodstream via nasal or lung mucosa, or diffuse directly into the olfactory nerves and pass onto the limbic system - a region associated closely with arousal (Hertz, 2009). Although the level of aromatic compounds that can be absorbed by these routes is low compared to other modes of administration (e.g., injection or ingestion), essential oils have been detected in the blood of rodents exposed to the vapours of essential oils (Jirovitz, Buchbauer, Jager, Raverdino, \& Nikiforov, 1990; Jirovitz, Buchbauer, Jager, Woiiieh, \& Nikiforov, 1992; Kovar, Gropper, Friess, \& Ammon, 1987). Currently, however, research has not produced data to suggest that inhaled volatiles become present in the bloodstream or in any other physiological system in humans - such a finding would support the notion that aromas have the intrinsic ability to interact with and affect human physiology at a very fundamental level. Future research 
would benefit by further investigating the pharmacological properties associated with various essential oils - distinct properties would imply substance-specificity, where a unique pattern of influence for any given aroma on cognitive domains and physiological parameters could be identified. To date, information is limited and further research is required if such properties and relationships are to be clearly identified.

\section{Conclusion}

In summary, this thesis contributes to the existing research regarding the differential effects of the inhalation of the essential oils of lavender and rosemary on arousal and cognitive performance. The results clearly demonstrate that certain aspects of cognitive performance and physiology can be influenced by exposure to these aromas. Some empirical substantiation regarding the purported sedating effects of lavender and stimulating effects of rosemary was presented; however, the notion of clear and opposing cognitive and physiological profiles for the aromas of lavender and rosemary received only mixed support. Ceiling effects observed across several cognitive tasks suggests that changes in cognitive performance due to ambient aroma exposure may be better studied using a battery of tasks specifically designed for this purpose. The disparity in the mood and physiological data brings into question the method of relying on self-report indices of arousal when assessing the physiological effects of scents on humans. This practice is predominant in the field of olfactory research, perhaps to the detriment of scientific pursuit. Although there is an acknowledged paucity of information in the field of olfactory research, it has quickly become an emerging field. The findings presented in this thesis query current research methodology and provide important contributions to the 
understanding of the effects of these scents in particular and, by extension, the potential for scents in general to have far-reaching influences on humans. 


\section{References}

Alaoui-Ismaili, O., Robin, O., Rada, H., Dittmar, A., \& Vernet-Maury, E. (1997). Basic emotions evoked by odorants: comparison between autonomic responses and self-evaluation. Physiology \& Behavior, 62(4), 713-720.

Bensafi, M., Rouby, C., Farget, V., Bertrand, B., Vigouroux, M., \& Holley, A. (2002). Autonomic nervous system responses to odours: the role of pleasantness and Arousal. Chemical Senses, 27, 703-709.

Bensafi, M., Rouby, C., Farget, V., Bertrand, B., Vigouroux, M., \& Holley, A. (2001). Are pleasant and unpleasant odors processed in the same way? Chemical Senses, 26, 786.

Bradley, M. M. \& Lang, P. J. (1999a). Affective norms for English words (ANEW): Instruction manual and affective ratings. Technical Report No.C-1, Center for Research in Psychophysiology, University of Florida, Gainesville.

Bradley, M. M. \& Lang, P. J. (1999b). International affective digitized sounds (IADS): Stimuli, instruction manual and affective ratings. Technical Report No. B-2, Center for Research in Psychophysiology, University of Florida, Gainesville.

Brauchli, P., Ruegg, P. B., Etzweiler, F., \& Zeier, H. (1995). Electrocortical and autonomic alteration by administration of a pleasant and an unpleasant odor. Chemical Senses, 20, 505-515.

Buchbauer, G., Jirovetz, L., Jager, W., Dietrich, H., \& Plank, C. (1991). Aromatherapy: Evidence for sedative effects of the essential oil of lavender after inhalation. $Z$ Naturforsch C., 46(11-12), 1067-1072. 
Burnett, K. M., Solterbeck, L. A., \& Strapp, C. M. (2004). Scent and mood state following and anxiety-provoking task. Psychological Reports, 95, 707-722.

Cacioppo, J. T. \& Tassinary, L. G. (1990). Inferring psychological significance from physiological signals. American Psychologist, 45(1), 16-28.

Cavanagh, H. M. A. \& Wilkinson, J. M. (2002). Biological activities of lavender essential oil. Phytotherapy Research, 16, 301-308.

Damian, P. \& Damian, K. (1995). Aromatherapy, scent and psyche: Using essential oils for physical and emotional well-being. Rochester: Healing Arts Press.

D'Angelo, R. (2002). Aromatherapy. In S. Shannon (Ed.), Handbook of complementary and alternative therapies in mental health (pp. 71-92). San Diego, CA: Academic Press.

Davis, R. G. (1977). Acquisition and retention of verbal associations to olfactory and abstract visual stimuli of varying similarity. Journal of Experimental Psychology: Human Learning and Memory, 3, 37-51.

Degel, J. \& Köster, E. P. (1999). Odors: implicit memory and performance effects. Chemical Senses, 24, 317-325.

Diego, M.A., Jones, N. A., Field, T., Hernandez-Reif, M., Schanberg, S., Kuhn, C., McAdam, V., Galamaga, R, \& Galamaga, M. (1998). Aromatherapy positively affects mood, EEG patterns of alertness and math computations. International Journal of Neuroscience, 96, 217-224. 
Ebert, U., Oertel, R., Wesnes, K. A, \& Kirch, W. (1998). Effects of physostigmine on scopolamine induced changes in quantitative electroencephalogram and cognitive performance. Human Psychopharmacology, 13, 199-210.

Hertz, R. S. (1998). Are odors the best cues to memory? A cross-modal comparison of associative memory stimuli. Annals of the New York Academy of Sciences, 855, 670-674.

Hertz, R. S. (2009). Aromatherapy facts and fictions: A scientific analysis of olfactory effects on mood, physiology and behavior. International Journal of Neuroscience, $119,263-290$.

Hongratanaworakit, T., Heuberger, E., \& Buchbauer, G. (2003). Human behavioural and physiological reactions to inhalation of sweet orange oil. Proceeds of the3rd World Congress on Medical and Aromatic Plants for Human Welfare. Chiangmai, Thailand, February 3-7, 455.

Hongratanaworakit, T. (2004). Physiological effects in aromatherapy. Songklanakarin Journal of Science and Technology, 26(1), 117-125.

Jerison, H. J. \& Pickett, R. M. (1963). Vigilance: a review and re-evaluation. Human Factors, 5, 211-238.

Kaye, J. (2004). Making scents: aromatic output for HCI. Interactions, 11(1), 49-61. Kennedy, D. O., Scholey, A. B., \& Wesnes, K. A. (2000). Dose dependent changes in cognitive performance and mood following acute administration of a ginseng to healthy volunteers. Nutritional Neuroscience, 4, 295-310. 
Kennedy, D. O., Scholey, A. B., \& Wesnes, K. A. (2001). Differential, dose dependent changes in cognitive performance and mood following acute administration of a gingko biloba/panax ginseng combination to healthy young volunteers. Nutritional Neuroscience, 4, 399-412.

Klemm, W. R., Lutes, S. D., Hendrix, D. V., \& Warrenburg, S. (1992). Topographical EEG maps of human responses to odors. Chemical Senses, 17(3), 347-361.

Kovar, K. A., Gropper, B., Friess, \& Ammon, H. T. P. (1987). Blood levels of 1, 8cineole and locomotor activity of mice after inhalation and oral administration of rosemary oil. Planta Medica, 53, 315-319.

Lang, P. J. (1995). The emotion probe: Studies of motivation and attention. American Psychologist, 50, 372-385.

Lang, P. J., Bradley, M. M, Fitzsimmons, J. R., Cuthbert, B. N, Scott, J. D., Moulder, B., \& Nangia, V. (1998). Emotional arousal and activation of the visual cortex: an fMRI analysis. Psychophysiology, 35, 199-210.

Lang, P. J, Greenwald, M. K, Bradley, M. M, Hamm, A. O. (1993). Looking at pictures: affective facial visceral and behavioral reactions. Psychophysiology, 30, 261273.

Lis-Balchin, M. (2006). Aromatherapy science: a guide to healthcare professionals. London: Pharmaceutical Press.

Ludvigson, H. W. \& Rottman, T. R. (1989). Effects of ambient odors of lavender and cloves on cognition, memory, affect and mood. Chemical Senses, 14(4), 525536.

McNair, D. M. \& Lorr, M. (1964). An analysis of mood in neurotics. Journal of abnormal social psychology, 69, 620-627. 
McNair, D. M., Lorr, M., \& Droppleman, L. F. (1971, 1992). Manual for the profile of mood states. San Diego: Educational and Industrial Testing Service.

Mehrabian, A. \& Russell, J. A. (1974). An Approach to Environmental Psychology. Cambridge, MA: MIT Press.

Michel Pozzo, A.C.2.i, Courbevoie, France.

Millot, JL., Brand, G., \& Morand, N. (2002). Effects of ambient odors on reaction time in humans. Neuroscience Letters, 322, 79-82.

Moss, M., Cook, J., Wesnes, K., \& Duckett, P. (2003). Aromas of rosemary and lavender essential oils differentially affect cognition and mood in healthy adults. International Journal of Neuroscience, 113(1), 15-38.

Motomura, N., Sakurai, A., \& Yotsuya, Y. (2001). Reduction of mental stress with lavender odorant. Perceptual and Motor Skills, 93, 713-718.

Olfacom Incorporated. (2009). A.C.2.i, Courbevoie, France.

O’Neill WM, Hanks GW, White L, Simpson P, Wesnes K (1995) The cognitive and psychomotor effects of opioid analgesics. I. A randomised controlled trial of single doses of dextropropoxyphrene, lorazepam and placebo in healthy subjects. European Journal of Clinical Pharmacology, 48, 447-453.

Philpott, C. M., Bennett, A., \& Murty, G. E. (2008). A brief history of olfaction and olfactometry. Journal of Laryngology and Otology, $122(7), 657-662$. 
Raudenbush, B., Koon, J., Smith, J., \& Zoladz, P. (2003). Effects of odorant administration on objective and subjective measures of sleep quality, postsleep mood and cognitive functioning, and alertness. North American Journal of Psychology, 5, 181-192.

Russell, J. A. (1980). A circumplex model of affect. Journal of Personality and Social Psychology, 39, 1161-1178.

Saeki, Y. \& Shiohara, M. (2001). Physiological effects of inhaling fragrances. International Journal of Aromatherapy, 11(3), 118-125.

Sakamoto, R., Minoura, K., Usui, A., Ishizuka, Y., \& Kanba, S. (2005). Effectiveness of aroma on work efficiency: lavender aroma during recesses prevents deterioration of work performance. Chemical Senses, 30, 683-691.

Scholey, A. B, Moss, M. C., Neave, N., \& Wesnes, K. A. (1999). Cognitive performance, hyperoxia and heart rate following oxygen administration in healthy young adults. Physiology \& Behavaviour, 67, 783-789.

Shepherd, G. M. (2004). The human sense of smell: Are we better than we think? PLoS Biology, 2, 572-575.

Shimizu, K., Gyokusen, M., Kitamura, S., Kawabe, T., Kozaki, T., Ishibashi, Izumi, R., Mizunoya, W., Ohnuki, K, \& Kondo, R. (2008). Essential oil of lavender inhibited the decreased attention during a long-term task in humans. Bioscience, Biotechnology and Biochemistry, 72(7), 1944-1947.

Simpson, P. M., Surmon, D. J.., Wesnes, K. A., \& Wilcock, G. K. (1991). The cognitive drug research computerized assessment system for dementia patients: A validation study. International Journal of Geriatric Psychiatry, 6, 95-102. 
Thought Technology Ltd. (2009). Montreal Canada. http://www.thoughttechnology.com.

Tisserand, R. (1977). The art of aromatherapy. Essex: C. W. Daniel.

Torii, S., Fakuda, H., Kanemoto, H., Miyanchi, R., Hamauzu, Y., \& Kawasaki, M. (1988). Contingent negative variation and the psychological effects of odor. In, S. Van Toller and G. H. Dodd (Eds.), Perfumery: The psychology and biology of fragrance. London: Chapman \& Hill, pp. 107-120.

United BioSource Corporation. (2009). Computerized cognitive testing: Setting the standard in cognitive assessment for drug research, Maryland: USA.

Van Toller, S. \& Dodd, G. H. (1992). Fragrance: the psychology and biology of perfume. London: Elsevier.

Warm, J. S., Dember, W. N., \& Parasuraman, R. (1991). Effects of olfactory stimulation on performance and stress in a visual sustained attention task. Journal of the Society of Cosmetic Chemists, 42, 199-210.

Warm, J. S., Dember, W. N, \& Hancock, P. A. (1996). Vigilance and workload in automated systems. In R. Parasuraman \& M. Mouloua (Eds.), Automation and human performance: theory and applications. Mahwah, NJ: Erlbaum, 183-200.

Watson, D., Clark, L. A., \& Tellegen, A. (1988). Development and validation of brief measures of positive and negative affect: The PANAS scale. Journal of personality and social psychology, 54, 1063-1070. 
Wesnes, K. A., Faleni, R. A., Hefting, N. R., Hoogsteen, G., Houben, J. J. G., Leonard, J., Petrini, O., \& van Lier, J. J. (1997). The cognitive subjective and physical effects of Ginkgobiloba/Panax ginseng combination in healthy volunteers with neuroasthenic complaints. Psychopharmacology Bulletin, 33, 677-683.

Wesnes, K. A., Ward, T., \& McGinty, A. (2000). The memory enhancing effects of Ginkgo biloba/Panax ginseng combination in healthy middle aged volunteers. Psychopharmacology, 152, 353-361.

Wolfe, N. \& Herzberg, J. (1996). Can aromatherapy oils promote sleep in severely demented patients? International Journal of Geriatric Psychiatry, 11, 926-927.

Yagyu, T. (1994). Neuropsychological findings on the effects of fragrance: lavender and jasmine. Integrative Psychiatry, 10, 62-67.

Yamaguchi, H. (1990). Effects of odor on heart rate. In The Psychophysiological Effects of Odor, Aromachology. Koryo, 168.

Zillmer, E. \& Spiers, M. V. (2001). Sensory-perceptual and motor systems. In Principles of Neuropsychology (pp.122-125). Belmont, CA: Wadsworth. 


\section{Appendix A. Informed Consent Form}

The purpose of an informed consent is to ensure that you understand the purpose of the study and the nature of your involvement. The informed consent has to provide sufficient information such that you have the opportunity to determine whether you wish to participate in the study.

Study Title: Cognitive Test Battery Evaluation and Influence on Arousal Levels.

Research Personnel:

Principal Investigator: Livia Sumegi, 1sumegi@connect.carleton.ca

Faculty Advisor: Dr. Gitte Lindgaard, 520.2600 ext. 2255 or gitte_lindgaard@carleton.ca

If you have any ethical concerns about this study, you may contact Dr. Monique Sénéchal (Chair of the Carleton University Ethics Committee for Psychological Research, 613-520-2600 x1155 or monique_senechal@carleton.ca). If you have any other concerns about this study, you may contact Dr. Janet Mantler (Chair of the Department of Psychology, 613-520-2600 x4173 or janet_mantler@carleton.ca).

Purpose: This research is being conducted for the purpose of evaluating a new cognitive test battery and its influence on physiological measures of arousal.

Task Requirements: In this study, you will be asked to complete questionnaires assessing mood and arousal as well as a task battery that includes various cognitive measures (e.g. memory, speed of attention). To gain a better understanding of your experience with the test battery, physiological indices will also be taken throughout the study including your heart rate, skin conductance, and electroencephalogram (EEG) recordings. The entire study will take approximately 60 minutes.

Payment: In compensation for taking part in this study, you will be awarded 1.0 Psychology course credits.

Potential Risk/Discomfort: Three forms of data will be gathered. One type will be psychometric, including questionnaires pertaining to mood, feelings of arousal, as well as demographic information. This component involves no potential physical or psychological risks. The second component involves performing various cognitive tasks and is also not expected to pose any risk to you physically or psychologically. The final form of data to be gathered will consist of physiological recordings, including electroencephalograms (EEG) to measure 'brain waves' along with measures of heart rate and skin conductance. EEG data are acquired through electrodes placed on the scalp, using very small amounts of gel while heart rate and skin conductance are 
measured using electrodes on your fingers. The physiological data component poses only minimal risk as all instrumentation used has International Organization for Standardization (ISO) 13485 and CE certification, indicating that it satisfies international standards for the design and manufacture of medical devices and has met consumer safety requirements. Slight discomfort from the use of conductive gel and/or tape for attaching electrodes may be incurred. If you feel anxious and/or are uncomfortable in any way about your involvement in this study, please bring your concerns to the researcher's attention.

Anonymity/Confidentiality: The information collected in this experiment will be kept anonymous and confidential. To ensure anonymity, all data will be coded such that your name is not associated with the data. Confidentiality will be assured by restricting data access to only research personnel directly involved with this experiment.

Right to Withdraw: You will be permitted to not answer any question(s) that you choose to omit. You have the right to withdraw from this experiment at any time without penalty. Your participation is completely voluntary.

I have read the above description of Cognitive Test Battery Evaluation and its Influence on Arousal and understand the conditions of my participation. I understand that certain facts about the study might be withheld from me, and the researchers might not, initially, tell full purpose of the study. However, the complete facts of the study will be revealed to me at the completion of the study session. My signature indicates that I agree to participate in this research study and this in no way constitutes a waiver of my rights:

Name of Participant

Date

I have explained the research to the participant and answered all of his/her questions. I believe that he/she understands the information described in this document and, with that understanding, freely and willingly consents to participate: 


\section{Appendix B. Pre-Experimental Questions}

Please answer the following questions to the best of your ability.

1. Gender:

$\square \quad$ Male

$\square \quad$ Female

2. Age:

3. Ethnicity:

$\square$ Caucasian

$\square$ African/African American

$\square$ Asian/Asian American

$\square$ Native American

$\square$ Hispanic/Latino

$\square \quad$ Other (please specify):

4. Are you fluent in English:

$\square$ Yes

$\square \quad$ No

5. Are you a smoker?

Yes

$\square \quad$ No

6. Do you have any allergies or chemical sensitivities?

$\square$ Yes

$\square \quad$ No

If yes, please specify: 


\section{Appendix C. CDR System Task Instructions

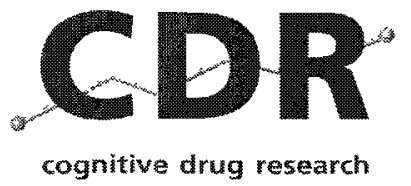

\section{Task Instructions for the}

\section{CDR System}

For all tasks, please ensure that the Participant responds as quickly as they can.

Each task is initiated by the administrator pressing the $<$ Enter $>\downarrow$ key once he/she is sure the Participant understands what to do. For the Spatial and Numeric Working Memory tasks, the space bar is used to redisplay the target stimuli, if necessary. Prior to each task (excepting the word and picture presentations) the administrator should always make sure that the Participant's fingers are resting gently on the appropriate response buttons so that the speed of the response can be precisely measured.

\section{The CDR System Task Battery}

\section{Word Presentation and Immediate Word Recall:}

Administrator: Please read the following instructions to the participant.

15 words will appear in the middle of the screen, one at a time. Try to remember as many as you can. After the last word has been shown, some instructions will appear on the screen informing you that you have 60 seconds to write down as many of the words as you can remember, in any order. Near the end of the test session, you will again be asked to recall as many of the words as you can remember, but without seeing them again. So it is important to get the words fixed firmly in your mind. Administrator: Once you are sure the participant understands the task, press the 'Enter' key to initiate the task.

\section{Picture Presentation:}

Administrator: Read the following instructions to the participant: 
This task measures your memory. A series of pictures will appear on the screen, one at a time. Please look at each picture carefully as it appears and try to remember as many as you can.

Administrator: Once you are sure the participant understands the task, press the $<$ Enter $>$ key to initiate the task.

\section{Simple Reaction Time:}

Administrator: Read the following instructions to the Participant:

This task measures your reaction time. You only need to use the YES button, so rest your finger gently on the YES button to get the fastest reaction time possible. The word YES will appear in the middle of the screen at varying intervals. Every time you see the word YES, press the YES button as quickly as you can.

Administrator: Once you are sure the Participant understands the task, press the $<$ Enter $>$ key to initiate the task.

\section{Digit Vigilance:}

Administrator: Read the following instructions to the Participant:

This task measures how well you can hold your concentration on a series of rapidly changing digits. You only need to use the YES button, so rest your finger gently on the YES button to get the fastest reaction time possible. A single digit will appear on the right-hand side of the screen and remain there.

Administrator: Press the $<$ Enter $>$ key to initiate this digit.

A continuous series of digits will appear in the middle of the screen. Every time the digit in the middle is the same as the digit on the right, press the YES button as quickly as you can, even if the digit in the middle has disappeared.

Administrator: Once you are sure the Participant understands the task, press the $<$ Enter $>$ key to initiate the task.

\section{Choice Reaction Time:}

Administrator: Read the following instructions to the Participant:

This task measures your reaction time. You will need to use both the YES and the NO buttons, so rest your fingers gently on the YES and NO buttons so you can get the fastest reaction time possible. Either the word YES or the word NO will appear in the middle of the screen at varying intervals. Every time you see the word YES, press the YES button 
as quickly as you can, and every time you see the word NO, press the NO button as quickly as you can.

Administrator: Once you are sure the Participant understands the task, press the $<$ Enter $>$ key to initiate the task.

\section{Spatial Working Memory:}

Administrator: Read the following instructions to the Participant:

This task measures how well you can remember where something was on the screen. You will see a house with 9 windows, 4 of the windows will be lit and 5 will be dark. You need to remember the position of the lit windows.

Administrator: Press the $<$ Enter $>$ key for the house to be shown on the screen. The house will remain on the screen for 10 seconds. The screen will then go blank. Now show the laminated diagram of the house to the Participant with the following instruction:

Can you point to the windows that were lit?

Administrator: If the Participant cannot point to the windows that were lit, press the 'space bar' to redisplay the house. The house can be redisplayed up to 2 times if required. Once you are sure that the Participant has remembered the position of the lit windows, continue with the task instructions:

The house will now appear again and again, but with only one window lit. For each house, if the window that is lit was also lit in the original house, press the YES button as quickly as you can, otherwise press the NO button as quickly as you can.

Administrator: Once you are sure the Participant understands the task, press the $<$ Enter $>$ key to initiate the task.

\section{Numeric Working Memory:}

Administrator: Read the following instructions to the Participant:

This task measures how well you can hold a short series of digits in your memory and how quickly you can recognize them. It is similar to remembering a telephone number for a short while. 5 digits will appear on the screen one at a time for you to remember. Say each digit aloud to help you remember it.

Administrator: Press the $<$ Enter $>$ key for the digits to be shown on the screen. After the digits have been shown, ask the Participant to repeat them back to ensure they remember them. If the Participant cannot remember the digits, press the 'space bar' to redisplay the 
digits. The digits can be redisplayed up to 2 times if required. Once you are sure that the Participant has remembered the digits, continue with the task instructions:

Now for each digit that appears on the screen, you should press the 'YES' button as quickly as you can if it is one of the digits you are remembering, or the 'NO' button if it is any other digit.

Administrator: Once you are sure the Participant understands the task and remembers the 5 digits, press the $<$ Enter $>$ key to initiate the next stage of the task.

\section{Delaved Word Recall:}

Administrator: Read the following instructions to the participant:

Now we are going back to the series of words you saw at the beginning of this testing session. You have 60 seconds to write down as many of the words that you can still remember. You can write down the same words that you wrote in the immediate word recall task, as well as any others you think were on the list.

Administrator: Once you are sure that the participant understands the instructions, press the $<$ Enter $>$ key to initiate the test

\section{Delayed Word Recognition:}

Administrator: Read the following instructions to the participant:

You will again see the series of words you saw at the beginning of the testing session one at a time on the screen. For each word, if you recognise it as being one of the words you saw at the beginning of the testing session, you should press the 'YES' button as quickly as you can, but if you do not recognise the word, you should press the 'NO' button as quickly as you can.

Administrator: Once you are sure the participant understands the task, press the $<$ Enter $>$ key to initiate the task.

\section{Picture Recognition:}

Administrator: Read the following instructions to the participant:

You will again see the pictures you saw at the beginning of the testing session mixed with similar pictures which will appear one at a time on the screen. For each picture, if you recognise it as 1 of those you saw at the beginning of the testing session, you should press the 'YES' button as quickly as you can, but if it is not one that you recognise you should press the 'NO' button as quickly as you can.

Administrator: Once you are sure the participant understands the task, press the $<$ Enter $>$ key to initiate the task. 
Appendix D. Arithmetic Computations

Block 1

96

39

11

65

$\times 3$

$\times 9$

$\times 9$

$\times 4$

$\times 9$

39

85

92

89

29

$+13$

$+75$

$+19$

$+31$

$+64$

$\longrightarrow$

$-$

$-$

44

40

99

89

59

$-36$

$-33$

$-43$

$-45$

$-40$ 
Block 2

\begin{tabular}{rrrr}
69 & 73 & 58 & 97 \\
$\times 7 \times 7$ & $\times 1$ & $\times 4$ & $\times 6$ \\
\hline & $\square \times$
\end{tabular}

\begin{tabular}{|c|c|c|c|c|}
\hline 19 & 83 & 75 & 29 & 92 \\
\hline 11 & +21 & +44 & +45 & +97 \\
\hline
\end{tabular}


Block 3

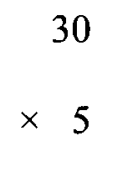

$\times 8$

$\times 8$

$\times 4$

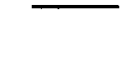

$+$

$\begin{array}{r}\times 8 \\ \hline\end{array}$

-

86

73

72

64

98

$+20$

$+13$

$+71$

$+56$

$-$

$\square+5 \square$

-

70

99

80

15

63

$\begin{array}{r}-39 \\ \hline\end{array}$

$-52$

$-44$

$-15$

$-59$ 
Block 4

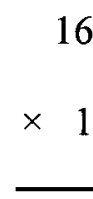

$$
9 \square
$$

49

99

16

$\times 1$

$7 \times 3$

$\times 2$

$\begin{array}{r}\times \\ \hline\end{array}$

$\begin{array}{r}\times 4 \\ \hline\end{array}$

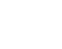

$\begin{array}{r}20 \\ +26 \\ \hline\end{array}$

86

56

12

58

$+85$

$+17$

$+54$

$+65$

-

-

-

$\begin{array}{r}74 \\ -12 \\ \hline\end{array}$

$\square \quad 70$

99

86

43

$-63$

$-30$

$-24$ 
Block 5

38

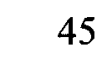

45

66

78

$\begin{array}{r}\times 3 \\ \hline\end{array}$

$\times 5$

$\times 1$

$\times 7$

$\times 6$

62

78

19

35

71

$+52$

$\square+2 \square$

$+89$

$+54$

$+52$

$\square \bar{\square}$

-

$-$

84

87

21

38

77

$-63$

$-37$

$-18$

$-24$

$-30$ 


\section{Appendix E. Post-Experimental Questions}

Please answer the following questions to the best of your ability.

1. Some conditions in this study included exposure to an aroma. If you believe you smelled a fragrance during this study, please rate the pleasantness of the scent you received. Circle your response.

$\begin{array}{ccccc}\text { Very } & \text { Somewhat } & \text { Neutral Somewhat } & \text { Very } \\ \text { Unpleasant } & \text { Unpleasant } & & \text { Pleasant } & \text { Pleasant } \\ 1 & 2 & 3 & 4 & 5\end{array}$

2. Please attempt to identify the scent

3. Are you currently experiencing seasonal allergies?

Yes

$\square$ No

4. Are you currently experiencing any cold or flu symptoms?
$\square$ Yes
No

5. In this study, we are concerned with the effects of scent on arousal and cognition - do you have any additional medical considerations that you feel may have affected your performance?

$\square$ Yes

$\square$ No

If yes, please elaborate: 


\section{Appendix F: Debriefing Form}

Study Title: Cognitive Test Battery Evaluation and Influence on Arousal Levels.

Thank you for participating in this study. Your time and effort are greatly appreciated.

The purpose of this study is to investigate the differential effects of fragrance inhalation of the essential oils of lavender and rosemary on arousal and cognitive performance. In this study, you were randomly placed in one of three conditions: lavender, rosemary, or no-scent (control). These two particular fragrances were chosen to be tested as they are believed to elicit opposite physiological reactions. The lavender-scented environment is expected to elicit a sedative effect whereas the inhalation of rosemary is expected to increase arousal levels. It is assumed that sedating odours such as lavender are more effective than stimulating odours for demanding/stressful tasks because of the anxiolitic effect they elicit. Conversely, stimulating odours such as rosemary are assumed to be more effective for less challenging or monotonous tasks as they help to maintain alertness. The cognitive test battery was actually administered for us to examine whether the aromas influence performance on the various cognitive domains tested.

In order to avoid the possibility of any expectancy effects contaminating the data, it was necessary to deceive you about the true purpose of the study. Please accept our apologies for that.

Minimal objective data exists regarding the impact of scents on human physiology and cognitive performance as olfaction (sense of smell) research is relatively new. This study aims to shed light on the ability of the aromatic chemicals of lavender and rosemary to alter mood, physiology and cognition. This research is designed to explore and highlight humans' unconscious vulnerability to olfactory stimuli.

Your time and effort are greatly appreciated.

Please feel free to contact us if you would to discuss any aspect of the research. The following people are involved in this study:

Dr. Gitte Lindgaard, Tel. 520.2600 ext.2255 or gitte_lindgaard@carleton.ca

Livia Sumegi, lsumegi@connect.carleton.ca

If you have any ethical concerns about this study please contact Dr. Monique Sénéchal (Co-chair, Dept. of Psychology Ethics Committee), (613) 520-2600 ext. 1155. For any other concerns, contact Dr. Janet Mantler, (Chair, Department of Psychology, Carleton University, 520-2600, ext. 4173).

Thank you again for being part of this important research! 
Appendix G. Consent to Use Data

$\mathrm{I}$, (full name), understand now that the purpose of the experiment was to learn more about the effects of scents on arousal and cognitive performance rather than to assist in the evaluation of cognitive measures. I also understand that it could have biased my performance if I had been informed about this in advance, which would have defeated the purpose of the experiment.

Now that I am aware of the real purpose of the experiment, I herewith give the researchers permission to use the data I have provided in this session.

Signed:

Date:

Witness:

Date: 


\section{Appendix H: ANOVA Summary Tables}

The following tables represent the analyses of variance that were conducted on the cognitive tasks and outcome factors, heart rate, skin conductance, and alpha and beta amplitudes.

H1. ANOVA summary table for reaction time performance on the Simple Reaction Time task by participant group

\section{ANOVA}

SRT
\begin{tabular}{|l|r|r|r|r|r|}
\hline & Sum of & & & & \\
& Squares & df & Mean Square & F & Sig. \\
\hline Between Groups & 18001.912 & 2 & 9000.956 & 5.257 & .007 \\
Within Groups & 143819.9 & 84 & 1712.141 & & \\
Total & 161821.8 & 86 & & & \\
\hline
\end{tabular}

H2. ANOVA summary table for reaction time performance on the Digit Vigilance task by participant group

\section{ANOVA}

VIGRT
\begin{tabular}{|l|r|r|r|r|r|}
\hline & Sum of & & & & \\
& Squares & df & Mean Square & F & Sig. \\
\hline Between Groups & 10227.579 & 2 & 5113.789 & 3.990 & .022 \\
Within Groups & 111514.4 & 87 & 1281.775 & & \\
Total & 121742.0 & 89 & & & \\
\hline
\end{tabular}

H3. ANOVA summary table for reaction time performance on the Spatial Working Memory task by participant group

\section{ANOVA}

SPMRT

\begin{tabular}{|l|r|r|r|l|l|}
\hline & \multicolumn{1}{|c|}{$\begin{array}{c}\text { Sum of } \\
\text { Squares }\end{array}$} & df & Mean Square & F & Sig. \\
\hline Between Groups & 442696.8 & 2 & 221348.400 & 3.605 & .032 \\
Within Groups & 4973201 & 81 & 61397.540 & & \\
Total & 5415898 & 83 & & & \\
\hline
\end{tabular}


H4. ANOVA summary table for performance on the Speed of Attention outcome measure by participant group

\section{ANOVA}

SpeedofAttention

\begin{tabular}{|l|r|r|r|r|r|}
\hline & \multicolumn{1}{|c|}{$\begin{array}{c}\text { Sum of } \\
\text { Squares }\end{array}$} & $\mathrm{df}$ & Mean Square & $\mathrm{F}$ & Sig. \\
\hline Between Groups & 86410.915 & 2 & 43205.457 & 3.242 & .044 \\
Within Groups & 1119589 & 84 & 13328.444 & & \\
Total & 1206000 & 86 & & & \\
\hline
\end{tabular}

H5. ANOVA summary table for performance on the Speed of Memory outcome measure by participant group

ANOVA

\begin{tabular}{|c|c|c|c|c|c|}
\hline & $\begin{array}{l}\text { Sum of } \\
\text { Squares }\end{array}$ & df & Mean Square & $\mathrm{F}$ & Sig. \\
\hline Between Groups & 2271888 & 2 & 1135943.754 & 3.845 & .025 \\
\hline Within Groups & 25700854 & 87 & 295412.112 & & \\
\hline Total & 27972741 & 89 & & & \\
\hline
\end{tabular}

H6. ANOVA summary table for mean heart rate by participant group

ANOVA

\begin{tabular}{|c|c|c|c|c|c|}
\hline & $\begin{array}{l}\text { Sum of } \\
\text { Squares }\end{array}$ & $\mathrm{df}$ & Mean Square & $\mathrm{F}$ & Sig. \\
\hline Between Groups & 1558.068 & 2 & 779.034 & $\overline{5.874}$ & .004 \\
\hline Within Groups & 11140.290 & 84 & 132.623 & & \\
\hline Total & 12698.358 & 86 & & & \\
\hline
\end{tabular}


H7. ANOVA summary table for task-related heart rate by participant group (condition), indicating a main effect for task-related heart rate and a main effect of condition.

Tests of Within-Subjects Effects

Measure MEASURE_1

\begin{tabular}{|ll|r|r|r|r|r|}
\hline Source & & $\begin{array}{c}\text { Type III Sum } \\
\text { of Squares }\end{array}$ & \multicolumn{1}{c|}{ df } & Mean Square & \multicolumn{1}{c|}{ F } & Sig \\
\hline HeartRate & Sphericity Assumed & 7377898 & 12 & 614825 & 17325 & 000 \\
& Greenhouse-Geisser & 7377898 & 4170 & 1769069 & 17325 & 000 \\
& Huynh-Feldt & 7377898 & 4520 & 1632261 & 17325 & 000 \\
& Lower-bound & 7377898 & 1000 & 7377898 & 17325 & 000 \\
\hline HeartRate * CONDITION & Sphericity Assumed & 1162020 & 24 & 48417 & 1364 & 113 \\
& Greenhouse-GeIsser & 1162020 & 8341 & 139314 & 1364 & 208 \\
& Huynh-Feldt & 1162020 & 9040 & 128541 & 1364 & 202 \\
& Lower-bound & 1162020 & 2000 & 581010 & 1364 & 261 \\
\hline Error(HeartRate) & Sphericity Assumed & 35771287 & 1008 & 35487 & & \\
& Greenhouse-GeIsser & 35771287 & 350322 & 102110 & & \\
& Huynh-Feldt & 35771287 & 379684 & 94213 & &
\end{tabular}

\section{Tests of Between-Subjects Effects}

Measure. MEASURE_1

Transformed Variable: Average

\begin{tabular}{|l|r|r|r|r|r|}
\hline Source & $\begin{array}{c}\text { Type III Sum } \\
\text { of Squares }\end{array}$ & df & Mean Square & \multicolumn{1}{|c|}{ F } & \multicolumn{1}{c|}{ Sig. } \\
\hline Intercept & 6965340.162 & 1 & 6965340.162 & 4258.357 & .000 \\
CONDITION & 14079.380 & 2 & 7039.690 & 4.304 & .017 \\
Error & 137397.717 & 84 & 1635.687 & & \\
\hline
\end{tabular}


H8. ANOVA summary table for heart rate for each task by participant group

ANOVA

\begin{tabular}{|c|c|c|c|c|c|c|}
\hline & & $\begin{array}{c}\text { Sum of } \\
\text { Squares }\end{array}$ & $\mathrm{df}$ & Mean Square & $F$ & Sig. \\
\hline WordPres_HR & $\begin{array}{l}\text { Between Groups } \\
\text { Within Groups } \\
\text { Total }\end{array}$ & $\begin{array}{r}1248.598 \\
17151.389 \\
18399.987\end{array}$ & $\begin{array}{r}2 \\
84 \\
86\end{array}$ & $\begin{array}{l}624.299 \\
204.183\end{array}$ & 3.058 & .052 \\
\hline ImmRecall_HR & $\begin{array}{l}\text { Between Groups } \\
\text { Within Groups } \\
\text { Total }\end{array}$ & $\begin{array}{r}765.977 \\
14696.259 \\
15462.235 \\
\end{array}$ & $\begin{array}{r}2 \\
84 \\
86\end{array}$ & $\begin{array}{l}382.988 \\
174.955\end{array}$ & 2.189 & .118 \\
\hline PicPres_HR & $\begin{array}{l}\text { Between Groups } \\
\text { Within Groups } \\
\text { Total }\end{array}$ & $\begin{array}{r}1012.919 \\
13681.813 \\
14694.732 \\
\end{array}$ & $\begin{array}{r}2 \\
84 \\
86 \\
\end{array}$ & $\begin{array}{l}506.460 \\
162.879\end{array}$ & 3.109 & .050 \\
\hline SimpleRT_HR & $\begin{array}{l}\text { Between Groups } \\
\text { Within Groups } \\
\text { Total }\end{array}$ & $\begin{array}{r}1177.285 \\
11442.201 \\
12619.486\end{array}$ & $\begin{array}{r}2 \\
84 \\
86\end{array}$ & $\begin{array}{l}588.642 \\
136.217\end{array}$ & 4.321 & .016 \\
\hline DigitVig_HR & $\begin{array}{l}\text { Between Groups } \\
\text { Within Groups } \\
\text { Total }\end{array}$ & $\begin{array}{r}901.140 \\
11040.290 \\
11941.429\end{array}$ & $\begin{array}{r}2 \\
84 \\
86\end{array}$ & $\begin{array}{l}450.570 \\
131.432\end{array}$ & 3.428 & .037 \\
\hline ChoiceRT_HR & $\begin{array}{l}\text { Between Groups } \\
\text { Within Groups } \\
\text { Total }\end{array}$ & $\begin{array}{r}746.858 \\
10758.797 \\
11505.655\end{array}$ & $\begin{array}{r}2 \\
84 \\
86\end{array}$ & $\begin{array}{l}373.429 \\
128.081\end{array}$ & 2.916 & .060 \\
\hline SpatialWM_HR & $\begin{array}{l}\text { Between Groups } \\
\text { Within Groups } \\
\text { Total }\end{array}$ & $\begin{array}{r}980.807 \\
12373.703 \\
13354.510 \\
\end{array}$ & $\begin{array}{r}2 \\
84 \\
86\end{array}$ & $\begin{array}{l}490.403 \\
147.306\end{array}$ & 3.329 & .041 \\
\hline NumWM_HR & $\begin{array}{l}\text { Between Groups } \\
\text { Within Groups } \\
\text { Total }\end{array}$ & $\begin{array}{r}933.658 \\
11985.225 \\
12918.883 \\
\end{array}$ & $\begin{array}{r}2 \\
84 \\
86\end{array}$ & $\begin{array}{l}466.829 \\
142.681\end{array}$ & 3.272 & .043 \\
\hline DelayedRecall_HR & $\begin{array}{l}\text { Between Groups } \\
\text { Within Groups } \\
\text { Total }\end{array}$ & $\begin{array}{r}864.508 \\
9757.898 \\
10622.407 \\
\end{array}$ & $\begin{array}{r}2 \\
84 \\
86\end{array}$ & $\begin{array}{l}432.254 \\
116.165\end{array}$ & 3.721 & .028 \\
\hline WordRec_HR & $\begin{array}{l}\text { Between Groups } \\
\text { Within Groups } \\
\text { Total }\end{array}$ & $\begin{array}{r}814.501 \\
9832.320 \\
10646.822 \\
\end{array}$ & $\begin{array}{r}2 \\
84 \\
86 \\
\end{array}$ & $\begin{array}{l}407.251 \\
117.051\end{array}$ & 3.479 & .035 \\
\hline PicRec_HR & $\begin{array}{l}\text { Between Groups } \\
\text { Within Groups } \\
\text { Total }\end{array}$ & $\begin{array}{r}674.501 \\
10252.576 \\
10927.077 \\
\end{array}$ & $\begin{array}{r}2 \\
84 \\
86 \\
\end{array}$ & $\begin{array}{l}337.250 \\
122.054\end{array}$ & 2.763 & .069 \\
\hline Math_HR & $\begin{array}{l}\text { Between Groups } \\
\text { Within Groups } \\
\text { Total }\end{array}$ & $\begin{array}{r}2576.009 \\
19149.203 \\
21725.212\end{array}$ & $\begin{array}{r}2 \\
84 \\
86\end{array}$ & $\begin{array}{r}1288.005 \\
227.967\end{array}$ & 5.650 & .005 \\
\hline
\end{tabular}


H9. ANOVA summary table for mean skin conductance by participant group

ANOVA

Mean_SC

\begin{tabular}{|l|r|r|r|r|r|}
\hline & \multicolumn{1}{|c|}{$\begin{array}{c}\text { Sum of } \\
\text { Squares }\end{array}$} & df & Mean Square & F & Sig. \\
\hline Between Groups & 15.702 & 2 & 7.851 & 1.201 & .306 \\
Within Groups & 529.685 & 81 & 6.539 & & \\
Total & 545.387 & 83 & & & \\
\hline
\end{tabular}

H10. ANOVA summary table for mean alpha and beta amplitudes by participant group

ANOVA

\begin{tabular}{|ll|r|r|r|r|r|}
\hline & \multicolumn{1}{|c|}{ Sum of } & Squares & \multicolumn{1}{c|}{ df } & Mean Square & F & Sig. \\
\hline AlphaAmplitudePower & Between Groups & 4313.961 & 2 & 2156.981 & 1.822 & .168 \\
& Within Groups & 98286.644 & 83 & 1184.176 & & \\
& Total & 102600.6 & 85 & & & \\
\hline BetaAmplitudePower & Between Groups & 568.964 & 2 & 284.482 & .614 & .543 \\
& Within Groups & 38438.239 & 83 & 463.111 & & \\
& Total & 39007.203 & 85 & & & \\
\hline
\end{tabular}


Appendix I: Comparison Table of CDR Outcome Variables by Participant Group

Effects of lavender and rosemary essential oils on individual task outcome measures from the CDR battery

\begin{tabular}{ccccc}
\hline $\begin{array}{c}\text { Outcome } \\
\text { Variable }\end{array}$ & $\begin{array}{c}\text { 1) Lavender } \\
\text { Mean } \pm \text { SD }\end{array}$ & $\begin{array}{c}\text { 2) Rosemary } \\
\text { Mean } \pm \text { SD }\end{array}$ & $\begin{array}{c}\text { 3) Control } \\
\text { Mean } \pm \text { SD }\end{array}$ & $\begin{array}{c}\text { Significant } \\
\text { comparisons }\end{array}$ \\
\hline $\begin{array}{c}\text { Simple Reaction } \\
\text { Time }\end{array}$ & $315.38 \pm 49.62$ & $285.27 \pm 39.73$ & $284.97 \pm 32.63$ & $\begin{array}{c}1 \text { vs } 2^{*} \\
1 \text { vs } 3^{*}\end{array}$ \\
$\begin{array}{c}\text { Digit Vigilance } \\
\text { Choice Reaction } \\
\text { Time }\end{array}$ & $427.68 \pm 37.63$ & $411.27 \pm 24.86$ & $398.32 \pm 35.88$ & 1 vs $3^{*}$ \\
$\begin{array}{c}\text { Spatial Working } \\
\text { Memory }\end{array}$ & $952.55 \pm 332.64$ & $827.88 \pm 214.57$ & $783.46 \pm 147.71$ & 1 vs 3* \\
$\begin{array}{c}\text { Numeric Working } \\
\text { Memory } \\
\text { Word }\end{array}$ & $805.98 \pm 163.25$ & $753.29 \pm 149.79$ & $769.59 \pm 147.99$ & \\
$\begin{array}{c}\text { Recognition } \\
\text { Picture }\end{array}$ & $868.47 \pm 185.02$ & $815.19 \pm 158.36$ & $808.61 \pm 115.19$ & \\
Recognition & $948.63 \pm 185.43$ & $937.99 \pm 177.59$ & $935.76 \pm 148.12$ & \\
\hline
\end{tabular}

